Humanités numériques

\title{
Une approche computationnelle du cadastre napoléonien de Venise
}

A Computational Approach to the Napoleonic Cadastre of Venice

Isabella di Lenardo, Raphaël Barman, Federica Pardini et Frédéric Kaplan

\section{OpenEdition}

Journals

Édition électronique

URL : https://journals.openedition.org/revuehn/1786

DOI : 10.4000/revuehn. 1786

ISSN : 2736-2337

Éditeur

Humanistica

Référence électronique

Isabella di Lenardo, Raphaël Barman, Federica Pardini et Frédéric Kaplan, « Une approche computationnelle du cadastre napoléonien de Venise », Humanités numériques [En ligne], 3 | 2021, mis en ligne le 01 mai 2021, consulté le 16 juillet 2021. URL : http://journals.openedition.org/revuehn/1786 ; DOI : https://doi.org/10.4000/revuehn.1786

Les contenus de la revue Humanités numériques sont mis à disposition selon les termes de la Licence Creative Commons Attribution 4.0 International. 


\title{
humanités numériques
}

$3 \mid 2021$

Humanités numériques spatialisées

INTÉGRATION ET USAGES DES DONNÉES HISTORIQUES ET PATRIMONIALES

\section{Une approche computationnelle du cadastre napoléonien de Venise A Computational Approach to the Napoleonic Cadastre of Venice}

\author{
Isabella di Lenardo, Raphaël Barman, Federica Pardini et \\ Frédéric Kaplan
}

\section{Résumés}

Au début du XIX ${ }^{\mathrm{e}}$ siècle, l'administration napoléonienne impose à la ville de Venise la mise en place d'un nouveau système de description standardisé pour rendre compte de manière objective de la forme et des fonctions du tissu urbain. Le cadastre, déployé à l'échelle européenne, offre pour la première fois une vue articulée et précise de la structure de la ville et de ses activités grâce à une approche méthodique et à des catégories standardisées. Les techniques numériques, basées notamment sur l'apprentissage profond, permettent aujourd'hui d'extraire de ces documents une représentation à la fois précise et dense de la ville et de ses habitants. En s'attachant à vérifier systématiquement la cohérence de l'information extraite, ces techniques évaluent aussi la précision et la systématicité du travail des arpenteurs et des sondeurs de l'Empire et qualifient par conséquent, de façon indirecte, la confiance à accorder aux informations extraites. Cet article revient sur l'histoire de ce protosystème computationnel, décrit la manière dont les techniques numériques offrent non seulement une documentation systématique, mais aussi des perspectives d'extraction d'informations latentes, encore non explicitées, mais implicitement présentes dans ce système d'information du passé.

At the beginning of the 19th century, the Napoleonic administration introduced a new standardised description system to give an objective account of the form and functions of the city of Venice. The cadastre, deployed on a European scale, was offering for the first time an articulated 
and precise view of the structure of the city and its activities, through a methodical approach and standardised categories. With the use of digital techniques, based in particular on deep learning, it is now possible to extract from these documents an accurate and dense representation of the city and its inhabitants. By systematically checking the consistency of the extracted information, these techniques also evaluate the precision and systematicity of the surveyors' work and therefore indirectly qualify the trust to be placed in the extracted information. This article reviews the history of this computational protosystem and describes how digital techniques offer not only systematic documentation, but also extraction perspectives for latent information, as yet uncharted, but implicitly present in this information system of the past.

\section{Entrées d'index}

MOTS-CLÉS : humanités numériques spatialisées, histoire, archives, numérisation, système d'information géographique, apprentissage automatique

KEYWORDS: spatial digital humanities, history, archives, digitisation, geographic information system, machine learning

\section{Introduction}

Les cadastres déployés à large échelle en Europe à partir du début du XVIII $^{\mathrm{e}}$ siècle représentent une source d'information géohistorique d'une grande pertinence pour les études historiques. Les techniques numériques d'extraction et d'analyse permettent non seulement de rendre compte de la richesse informationnelle de ces sources administratives mais de constituer des bases de données combinant des informations précises sur la population avec des indications géométriques sur les villes et les territoires (Noizet, Bove et Costa 2013; Micalizzi, Buonora et Sasso D'Ellia 2010 ; Bianchi et Vangelisti 2014). Cet article présente les recherches effectuées depuis plusieurs années sur le cadastre napoléonien établi en 1808 pour la ville de Venise. Dans la continuité de publications sur les méthodes d'extraction semi-automatique des formes géométriques dans ce type de document historique (Ares Oliveira, di Lenardo et Kaplan 2017 ; Ares Oliveira et al. 2019), cet article explore les types d'analyse et de visualisation qui peuvent être produits sur le tissu urbain et la population de Venise dans les années qui suivent la chute de la République.

L'article débute sur une remise en contexte historique du document étudié et sur une discussion des modalités pratiques de sa production. Il est en effet crucial, pour interpréter correctement le contenu administratif du document, d'associer à l'extraction des données la remise en perspective d'un processus logistique impliquant des standards et des techniques établis dans un contexte historique et politique particulier. Létablissement du cadastre répond dans ce cas à une volonté administrative spécifique, liée à un nouveau calcul des recettes et dépenses de la 
ville. Par ailleurs, le cadastre vénitien de 1808, même s'il donne l'illusion de capturer un instantané historique, une photographie synchronique de l'état de la ville sur une année donnée, résulte en fait pragmatiquement d'un processus d'enquête, de comptage, de correction et de documentation qui s'étale sur une durée beaucoup plus longue. La reconstruction précise de l'histoire de la production du document luimême et la mise en relation de cette information avec les données extraites est une condition nécessaire de leur interprétabilité et de leur articulation avec d'autres jeux de données qui pourraient être produits à la même époque.

L'article décrit ensuite le cadastre comme un protosystème informationnel dont il convient non seulement de modéliser le contenu, mais aussi d'évaluer la cohérence. Nous décrivons une approche heuristique basée sur une liste de contraintes qui tente, d'une part, de corriger d'éventuelles erreurs résultant du processus semi-automatique de redocumentation, d'autre part, d'estimer la cohérence intrinsèque des informations produites par l'administration vénitienne appliquant la méthode napoléonienne. Cette estimation a posteriori, probablement inédite pour un document de cette ampleur, permet de produire un jeu de données historiques consolidé et offre une contextualisation de la précision d'un processus administratif datant d'il y a plus de deux cents ans.

L'article se conclut sur une première exploration géohistorique des structures que révèle ce document. Il s'agit de rendre compte de l'entremêlement spatial des fonctions de la ville et de l'articuler avec les typologies du bâti. L'analyse spatiale de la population elle-même, ainsi que les informations sur la propriété foncière des grandes familles vénitiennes, ne sont pas abordées ici et seront traitées dans un autre article. L'article illustre par contre la manière dont les informations géohistoriques sur la spatialité des fonctions de la ville peuvent donner lieu à des représentations dérivées, appelées ici cartes proxémiques, qui démontrent l'existence d'une information latente, non directement lisible dans les registres et plans de l'administration, mais qui peut être révélée par des visualisations calculées. Le cadastre napoléonien redocumenté par les techniques numériques devient à son tour la source de nouvelles cartographies de la ville du passé. 


\section{La ville calculée}

\section{L'introduction du cadastre napoléonien à Venise}

Une transformation importante de la classification administrative commence en France après la révolution de 1789. Très vite, à partir de la création des départements, les entités administratives fonctionnelles sont classées selon des principes protostatistiques dans lesquels, par exemple, la taille de la population est déterminante. Au sein de la population, l'analyse des catégories sociales et des niveaux de production est cruciale pour déchiffrer des systèmes urbains complexes et définir des stratégies de gestion de l'urbanisme. Cette transformation numérique des hiérarchies fonctionnelles et administratives s'éloigne définitivement de la perception symbolique et iconographique de l'espace urbain qui dominait sous l'Ancien Régime. En d'autres termes, les villes mesurent dès lors leurs importances respectives en fonction des catégories statistiques et quantitatives liées à la taille de la population (Roncayolo 1987) et à la richesse effectivement acquise par le biais de l'actif fiscal, et non plus en fonction de l'aura d'un passé illustre et mythique qui aurait marqué l'histoire, de la présence d'un siège épiscopal ou des forces militaires dont elles disposent.

Venise, resplendissante et grandiose depuis des siècles en tant qu'entité immuable de l'aristocratie citadine, fait partie intégrante depuis 1806 du système politico-administratif du royaume d'Italie, qui est soumis au contrôle strict de Paris par l'intermédiaire de la capitale, Milan. Les Français font de l'État italien un homologue formel du modèle français, dans lequel s'applique une subdivision en départements et en chefs-lieux sur la base de la taille de la population (Mascilli Migliorini 2011). La République vénitienne doit donc abdiquer définitivement son rôle dominant - elle était appelée la " Dominante " - sur les villes voisines telles que Padoue, Trévise, Vicence et Vérone, qu'elle avait soumises par la force au cours des siècles (Zucconi 2002).

Ce fut pour Venise une perte de dignité. La ville passe, comme le mentionnent certaines métaphores littéraires de l'époque, du statut de ville dominante à celui de ville soumise, et se retrouve dans une position de subordination par rapport à Milan, Florence et Rome, avec lesquelles elle rivalisait encore une décennie plus tôt (Antonielli 2001). La République de Venise a de facto abandonné son ordre étatique et partage désormais avec les autres villes voisines le statut juridique de municipalité.

Pour officialiser ce changement historique, l'administration française charge quelques fonctionnaires italiens de procéder à un immense travail d'analyse statistique (Rossi 1998). Suivant une hiérarchie des tâches bien définie, tous les organes de l'État sont mobilisés pour tenter d'appliquer les classifications de l'administration napoléonienne au contexte italien, en essayant, comme dans le cas de l'analyse cadastrale, de les adapter aux catégories interprétatives locales qui, pendant des siècles, avaient structuré l'analyse des structures urbaines. Les préfets de chaque département doivent, d'une part, produire des relevés précis du territoire, sans aucune distinction méthodologique entre les espaces urbains 
et ruraux, et, d'autre part, collecter les questionnaires établis pour chaque entité territoriale afin de recueillir, autant que possible, des données classées et homogènes par structure.

Le travail de collecte de données se basant sur des catégories standardisées peut être décrit comme une sorte d'inventaire raisonné et homogène de la géographie des objets urbains et non urbains. Il s'agit d'une d'application de l'interprétation du territoire selon des normes urbanistiques non encore théorisées de manière cohérente, mais qui sont le fruit d'observations pragmatiques et d'approches interprétatives typiques de structures militaires habituées au caractère hétérogène de l'information géographique.

Entre 1806 et 1809, Melchiorre Gioia est chargé par l'administration française de produire une enquête sur les centres de population (Capra 1978). Gioia, directeur du nouvel Office des statistiques, ne dispose pas encore d'une méthodologie cohérente pour la collecte et l'interprétation de données quantitatives (Gioia 1808), mais entame un travail pionnier qui a donné naissance à l'Institut national des statistiques de l'Italie unifiée (Patriarca 1996). L'introduction de variables quantitatives comme clé pour l'analyse des données urbaines marque une révolution dans la subdivision fiscale (Bourguet 1988) et motive l'adoption du cadastre parcellaire à une si grande échelle. L'introduction du cadastre, imposée par l'administration française, répond en effet d'un point de vue théorique et pragmatique à la nécessité idéologique de répartir la contribution fiscale de manière plus équitable, suivant un objectif de proportionnalité en nette rupture avec l'arbitraire et l'irrégularité qui régnaient avant son introduction. Elle répond également, dans le seul intérêt français, à la nécessité de régler les dettes antérieures contractées par l'État, qui contribuèrent en partie à alimenter la Révolution de 1789 .

Le registre foncier doit servir de base pour un calcul équitable de la répartition de l'impôt. Il a été calculé qu'en 1796-1797 la contribution fiscale prélevée sur les territoires conquis - neuf départements belges et quatre départements de Savoie - permit de soulager la France de 26 millions de francs. L'impôt fut fixé à $1 / 6$ du revenu foncier les années suivantes (Clergeot 2007).

\section{L'établissement du plan}

L'année 1806 constitue l'étape initiale de la mise en place des principales dispositions administratives et cadastrales pour la ville de Venise. L'année suivante, la ville fait partie des chefs-lieux dont la fiscalité est redéfinie uniquement sur la base de critères quantitatifs. Avant ces transformations, les recettes municipales n'étaient pas basées sur un impôt individuel, mais uniquement sur la taxation des échanges. Ce système permettait à Venise de combler son déficit budgétaire avec les recettes provenant des innombrables taxes commerciales, notamment celle sur le transport par eau. D'autre part, Venise, en tant que capitale d'un État, avait une gestion budgétaire propre. Elle n'avait ni dépenses ni recettes municipales au sens strict. Tous les mouvements comptables étaient affectés à l'État dans son ensemble (Doria 2014).

Les dépenses de la ville de Venise sont estimées à 700 ooo lires. Les Français introduisent donc la taxe dite casatico pour faire face à ces dernières et tenter de faire de Venise une ville autonome d'un point de vue 
financier. L'objectif était de taxer les revenus locatifs de près de 4 ooo maisons et de vendre les propriétés de l'État pour une valeur de 6 millions de lires, afin de combler les dettes.

À ces fins, une commission assistée par l'architecte Giannantonio Selva est créée en 1807 (Romanelli 1988, 42) pour dresser une carte sur laquelle sera dessinée la nouvelle structure de la ville, redéfinissant de nouveaux espaces publics qui résultent eux-mêmes d'une vaste campagne d'expropriation. Simultanément, on trace un relevé qui conduira à la création des différentes planches du cadastre, appelé désormais napoléonien (figure 1). Pour réaliser ce nouveau plan géométrique, une série de normes sont donc publiées. L'objectif est de codifier une restitution correcte des objets géographiques et urbains avec une même grammaire graphique en termes d'échelle et de couleurs.

FIGURE 1. RECONSTITUTION DU MAILLAGE DU CADASTRE NAPOLÉONIEN DE VENISE APRÈS GÉORÉFÉRENCEMENT DES PLANCHES

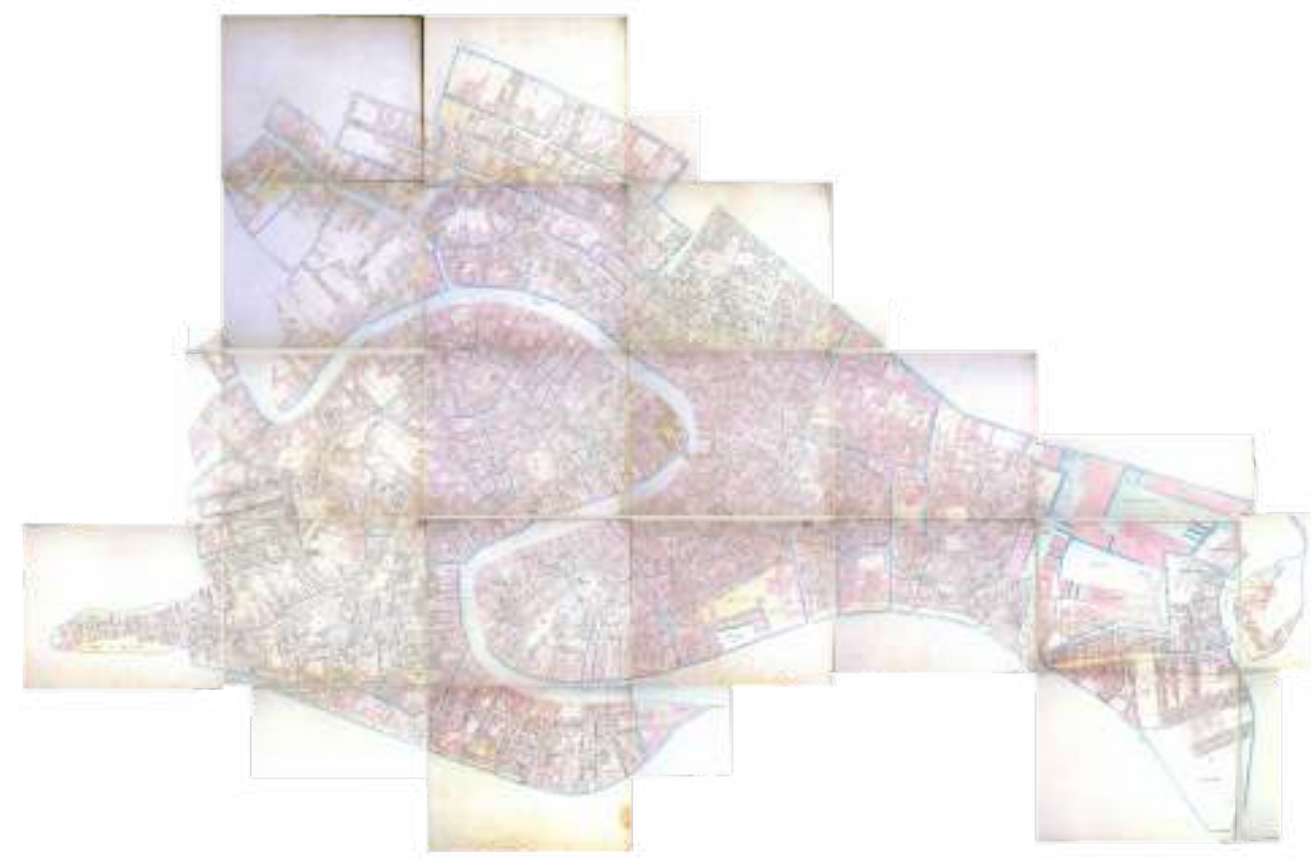

(C) EPFL - DHLAB

Dans la même période, Eugène de Beauharnais ordonne un décret (Decreto $n .62,1807$ ) qui esquisse les principales lignes directrices en définissant l'unité de mesure et les outils à utiliser, et établit l'usage des registres fonciers correspondants. La rédaction de cette étude constitue le premier relevé cartographique systématique de l'ensemble de la ville.

Historiquement la République de Venise n'avait jamais produit de carte unitaire, à l'exception du plan gravé par Ludovico Ughi en 1729, dont le réalignement géographique, par le biais du géoréférencement, a toutefois montré qu'il ne correspond pas à un relevé précis et qu'il contient d'importantes erreurs en termes de proportions de l'espace. Cette carte a probablement servi à plusieurs reprises au cours du XVIII ${ }^{\mathrm{e}}$ siècle pour gérer la structure urbaine, et constitue encore un dessin d'appui au début des années 1800 lorsque Giannantonio Selva l'utilise comme base cartographique pour le nouveau dessin de la ville. L'enquête introduite avec la méthode imposée par le nouveau gouvernement français génère une série de cartes complètement nouvelles, avec un niveau de confiance cartographique bien plus élevé. 
Depuis 1804, le gouvernement français a défini une méthode de relevé et de documentation sous forme de cartes et de registres. Les instructions concernent les relevés, qui doivent obéir à des règles précises et homogènes pour éviter toute décision arbitraire des arpenteurs et toute pression sur les personnes sondées. Le processus est décrit à plusieurs reprises dans des manuels qui convergent, en 1811, dans un ouvrage clé, le Recueil méthodique des lois, décrets, règlements, instructions et décisions sur le cadastre de la France.

On peut établir quelle méthodologie utilisaient les géomètres vénitiens grâce à un ouvrage s'adressant directement aux ingénieurs chargés de réaliser le levé puis l'estimation du territoire. Publiée en italien en 1810, la première édition des Istruzioni per i geometri (Repele, Rossi et Tonetti 2011) rassemble déjà des directives plus précises par rapport au décret royal institué trois ans plus tôt. L'ouvrage contient les prescriptions spécifiques pour le mesurage des terrains et la délimitation des cartes et des registres annexés. Publié dans un format de poche, il peut accompagner le géomètre dans son travail quotidien (Repele, Rossi et Tonetti 2011, 34). C'est une sorte de vade-mecum invitant à suivre strictement ses indications et définissant les instruments essentiels pour rendre universelles les opérations de taxation.

La rédaction des cartes proprement dite est précédée par la délimitation et la subdivision du territoire municipal en sections morphologiquement ou économiquement homogènes (identifiées par des majuscules et par des toponymes sur le dessin), ce qui nécessite une délicate appropriation et négociation bureaucratique.

Pour leur restitution géométrique, les plans suivent les canevas de construction trigonométrique (Carassi et Massabò Ricci 1987, 105). Même si elles sont divisées en différentes planches, les cartes doivent représenter une seule municipalité à la fois, avec le nom des villes limitrophes écrit en majuscules au-delà des frontières dessinées.

Des instructions sur les couleurs et les techniques de hachure sont données pour tout élément à représenter afin d'établir une grammaire visuelle spécifique, illustrée dans la figure 2. Cette dernière permet ainsi de mettre en évidence chaque élément imposable : environnement bâti, places, champs, routes, ponts, voies navigables, ports, falaises.

FIGURE 2. GRAMMAIRE VISUELLE DES ÉLÉMENTS GRAPHIQUES CONSTITUANT LES PLANCHES DU CADASTRE NAPOLÉONIEN
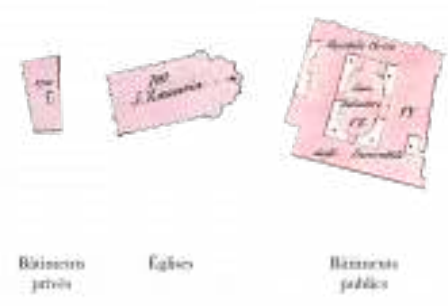
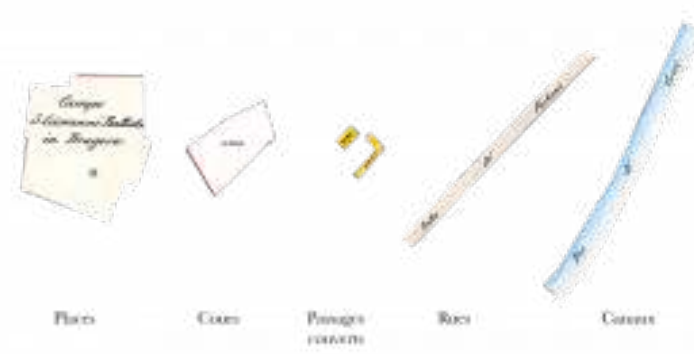
Une fois terminée, la carte est affichée à l'hôtel de ville pendant quarante jours pour garantir le droit de contester des erreurs potentielles ou des descriptions erronées (Locatelli 2003).

La carte est complétée par un registre foncier appelé sommarione, présenté dans la figure 3. Il correspond à la liste des personnes imposables liées à la représentation planimétrique. Pour chaque parcelle, les propriétaires sont mentionnés ainsi que la description des différents terrains et bâtiments. La méthode et les catégories standardisées de description sont construites pour laisser peu de marge d'interprétation et éviter les erreurs lors de la mise en commun des recensements. L'estimation des surfaces des propriétés cartographiées a lieu après l'arpentage, une fois tous les relevés compilés.

FIGURE 3. PAGE D'UN SOMMARIONE

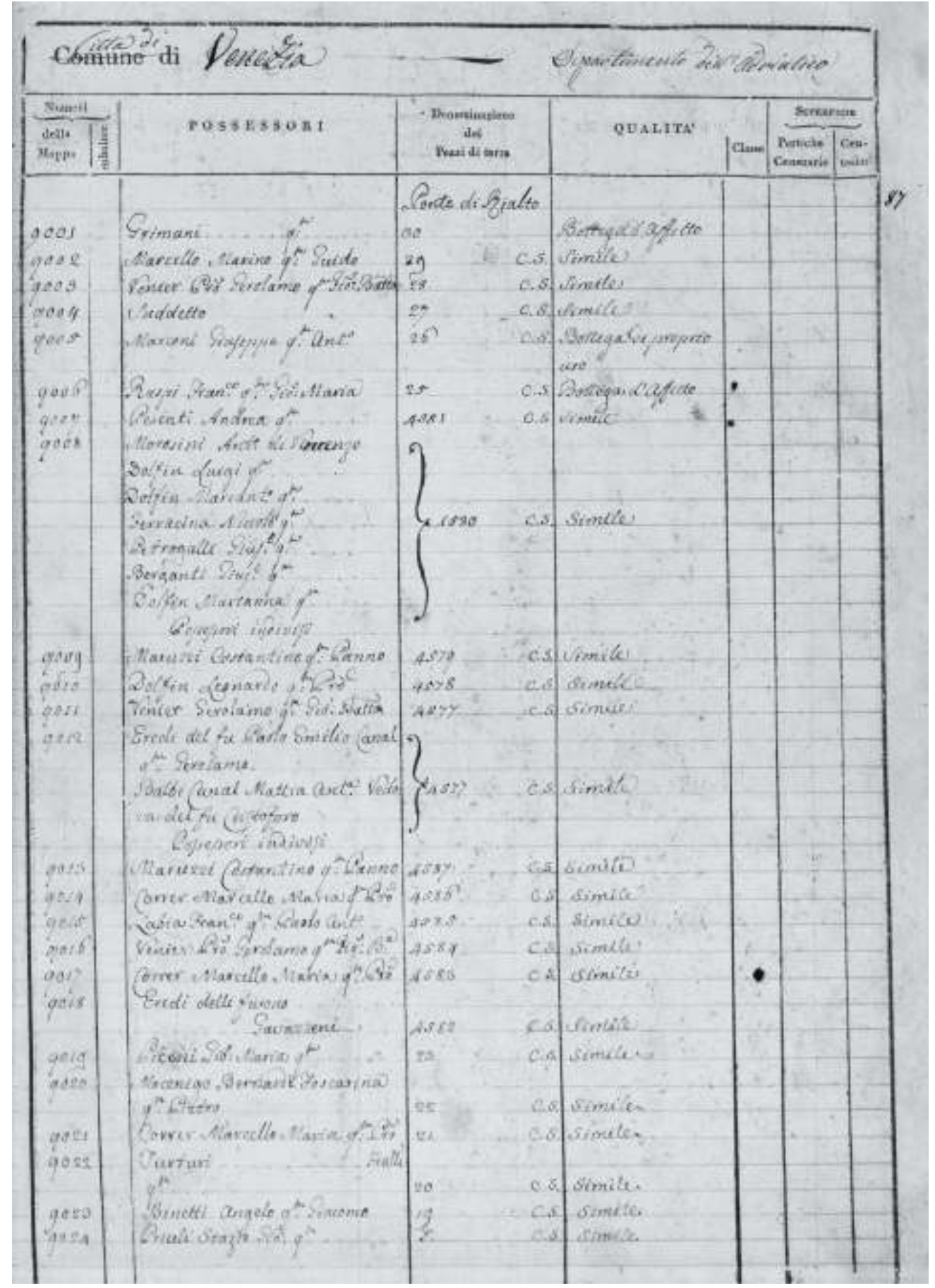

Document des archives d'État de Venise, fonds Archivio di Stato di Venezia, Censo Stabile, Catasto Napoleonico.

(C) EPFL - DHLAB 


\section{Le cadastre napoléonien comme protosystème d'information urbaine}

\section{La structure des données}

Le cadastre napoléonien se compose de trois documents interdépendants qui constituent le système d'information (Kain et Baigent 1992) :

- Le premier est le Plan cadastral, c'est-à-dire l'atlas qui est composé de plusieurs feuilles (planches) représentant la carte, qui suit grammaire visuelle précise et identifie avec des numéros les objets présents sur le territoire qui doivent être taxés. L'échelle de la carte peut varier de $1 / 500$ à $1 / 2$ ooo dans le cas des centres urbains, atteignant 1/5 ooo dans le cas des champs ou des territoires plus vastes.

- Le deuxième document est le registre connu en France sous le nom d'État de sections, qui contient toutes les parcelles par ordre croissant avec quelques informations de base.

- Le troisième, index inversé du second, est la matrice cadastrale, qui structure les informations par nom de propriétaire.

L'application de cette structure générale au cas de Venise (fonds Archivio di Stato di Venezia, Censo Stabile, Catasto Napoleonico) conserve les mêmes éléments constituants (Pavanello 1981). Comme résumé sur la figure 4, la structure de l'information est donc constituée d'une carte (mappale) qui est le squelette visuel de la représentation du système des propriétaires, des droits et de la fiscalité. Chaque numéro de parcelle joue le rôle d'une clé d'identification pour le registre de l'État de sections (sommarione) qui permet d'accéder à des informations complémentaires. Dans la matrice cadastrale, un identifiant unique est attribué à chaque propriétaire dans l'État de sections. Toutes les propriétés et données fiscales d'un propriétaire sont ainsi résumées.

FIGURE 4. STRUCTURE DES RENVOIS ENTRE LES DOCUMENTS QUI COMPOSENT LE SYSTĖME D'INFORMATION DU CADASTRE NAPOLÉONIEN

Doc. I Plan cadastral

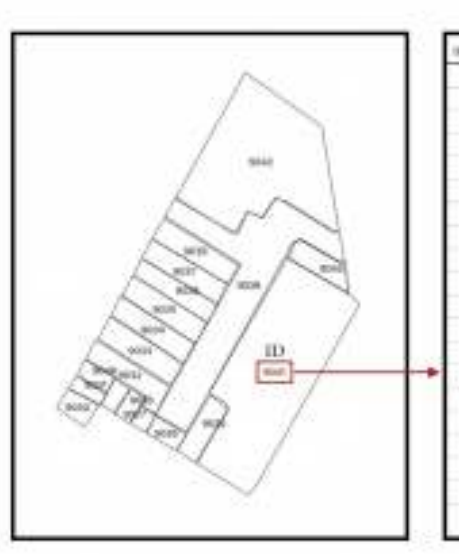

(c) EPFL - DHLAB

- DH
Doc. 2 État de sections (sommarione)

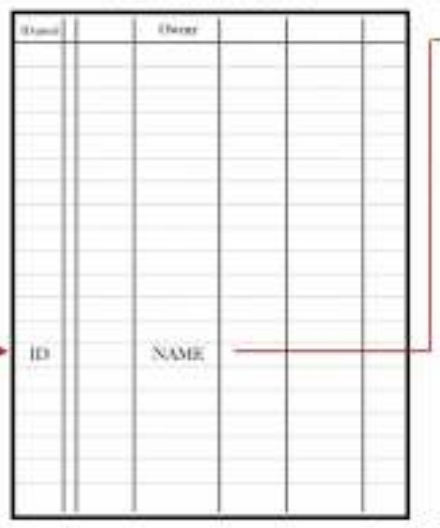

Doc. 3 Matrice cadastrale

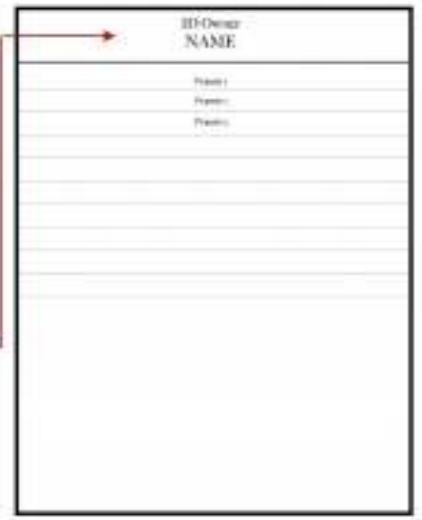


Les sommarioni doivent contenir toutes les informations utiles pour constituer un système d'information fiable permettant une consultation rapide et ayant tous les éléments pour procéder à une imposition qui tienne compte des catégories de biens possédés et de leurs surfaces. Il doit contenir pour chaque bien son propriétaire, sa catégorie et des informations permettant de l'identifier de manière non ambiguë. Les différents champs du sommarione sont présentés dans la figure 5.

FIGURE 5. STRUCTURE DES CHAMPS DANS LE SOMMARIONE

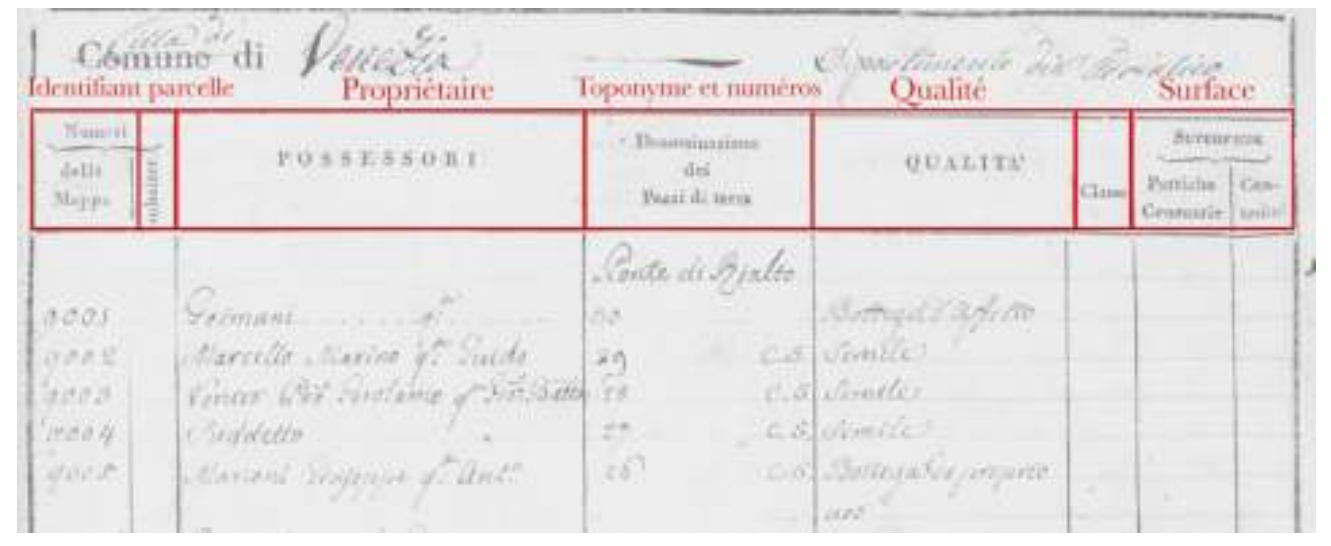

(c) EPFL - DHLAB

La parcelle cadastrale est représentée par un identifiant unique $(\mathrm{Nu}$ meri della mappa), qui croît de manière progressive pour relier les numéros de la carte à la propriété dans le registre. Pour les toponymes (Denominazione dei pezzi di terra), les descriptions textuelles utilisées dans les systèmes fiscaux précédents (Chauvard 2007) sont remplacées par une segmentation standardisée de l'espace reposant sur des toponymes identiques dans le registre (sommarione) et sur la carte (mappale). Pour rendre l'identification de la parcelle encore plus précise, l'administration française introduit la numérotation des logements. L'introduction de la numérotation est nécessaire, car il peut y avoir une parcelle de terrain sur laquelle se trouvent plusieurs unités et plusieurs propriétaires. Alors que l'identification du numéro de parcelle est unique, progressive pour toute la ville, et qu'il ne peut y avoir deux fois le même numéro, la numérotation des unités de logement recommence dans chaque sestiere (les sestieri sont au nombre de six et leurs frontières sont encore les mêmes aujourd'hui). Le champ subalterno est utilisé pour indiquer la coexistence de plusieurs unités de logement et de plusieurs propriétaires sur la même parcelle. La présence de plusieurs subalterni dénote une fragmentation de la propriété au sein d'une même parcelle cadastrale.

L'introduction de toutes ces spécifications - toponyme, numéro et $s u$ balterno - répond à l'exigence de réduire la complexité d'un objet en 3D, qui se développe en hauteur, avec différents propriétaires, à une description en $2 \mathrm{D}$ sur la carte.

Le champ de description du propriétaire (possessore) indique un ou plusieurs propriétaires par parcelle. Les rédacteurs du document ont tenté d'uniformiser la grammaire de la description des propriétaires. Le signe distinctif de la nouvelle politique, après l'abolition des privilèges aristocratiques, est la suppression du préfixe Nobile huomo, devant les noms des anciennes familles nobles vénitiennes et du préfixe Messere devant les lignées sans titre. Tous les propriétaires ont maintenant le 
même statut social de citoyen. Pour garantir l'identification au sein de la généalogie, le quondam, ou patronyme du propriétaire, reste néanmoins utilisé.

Le champ " qualité " (qualità) correspond à la description fonctionnelle de la parcelle ainsi qu'au droit d'utilisation qui lui est associé. Il s'agit d'un champ dense dans lequel les rédacteurs ont défini des classes d'objets d'un point de vue fonctionnel et non descriptif, et des classes d'utilisation, essentiellement l'emplacement ou l'usage personnel du bien. C'est précisément de cet ensemble d'informations que nous traitons dans cet article.

\section{Extraire et vérifier l'information}

Les méthodes numériques d'analyse permettent de redocumenter les informations contenues dans ce type de documents sous la forme de bases de données. Ce travail de rétro-ingénierie permet non seulement de transformer potentiellement ces documents en "Big Data du passé » (Kaplan et di Lenardo 2017) mais aussi d'évaluer la précision intrinsèque de ces informations compilées il y a plus de deux cents ans. Chaque numéro de parcelle de la carte est-il véritablement associé à une entrée dans les sommarioni ? Inversement, chaque entrée des sommarioni correspond-elle à une parcelle sur la carte ? Y a-t-il des doublons ou au contraire des lacunes dans la numérotation parcellaire ? Vérifier ces éléments et évaluer la qualité globale du système informationnel produit permet de donner une mesure quantitative de l'efficacité des processus mis en place par l'administration française et symétriquement de produire une première évaluation de la confiance que l'on peut accorder aux informations que ces documents contiennent.

\section{Le plan cadastral}

Le plan cadastral est composé de vingt-sept planches, ce qui correspond à plus de 23000 parcelles. Chaque planche a été numérisée par les archives d'État de Venise au format TIFF. Les images ont ensuite été géoréférencées, dans le cadre de notre recherche, sur le plan du système d'information géométrique vectoriel de la ville contemporaine fourni par la mairie de la ville de Venise. Le géoréférencement a été réalisé à l'aide de l'outil de géoréférencement du logiciel open source QGIS. Pour chaque planche, entre 5 et 7 points de calage ont été choisis en évitant les ponts, les quais et les réseaux routiers susceptibles de ne pas constituer des amers stables dans le temps et en privilégiant les angles des principaux bâtiments historiques. La transformation géographique utilisée est de nature " affine " puisque le raster de départ n'est pas courbé ou incurvé ${ }^{1}$. Les planches géoréférencées ont été enregistrées au format GeoTIFF, qui permet d'ajouter des informations de géoréférencement à une image TIFF. Un réseau d'images combinant l'ensemble des planches géoréférencées, appelé tableau d'assemblage, est ainsi constitué pour permettre l'extraction automatique des géométries des parcelles ainsi que de leurs numéros d'identification pour faire le lien avec les registres.

En 2017, une première étude sur l'extraction du cadastre napoléonien de Venise a montré des résultats prometteurs en matière d'extraction des parcelles et de reconnaissance des identificateurs. Cependant, cette méthode était entièrement conçue comme un pipeline ad hoc, adapté à 
un cadastre particulier (Ares Oliveira, di Lenardo et Kaplan 2017). La méthode utilisée dans cette étude est plus générique. Elle fait usage de l'apprentissage profond (deep learning) afin de segmenter les images du plan cadastral pour identifier les bordures des parcelles et les segments de texte (Ares Oliveira et al. 2019). Les autres méthodes utilisant l'apprentissage profond pour la segmentation de cartes soit sont moins performantes que des méthodes plus simples (Karabork et al. 2008), soit ne sont pas utilisées pour traiter des plans cadastraux et ne permettent donc pas d'extraire les géométries parcellaires (Uhl et al. 2020 ; Chiang et al. 2020).

Le réseau de segmentation utilisé est un réseau neuronal entièrement convolutif inspiré de l'architecture U-Net (Ronneberger, Fischer et Brox 2015). Un réseau ResNet-50 (He et al. 2016) est utilisé comme encodeur. Ce réseau est pré-entraîné sur le jeu de données Imagenet (Deng et al. 2009), ce qui permet d'accélérer son entraînement, réduit la quantité de données nécessaires et aide à la généralisation. Les détails de cette architecture générique sont développés dans l'article d'Ares Oliveira, Seguin et Kaplan (2018).

Le réseau de neurones est entraîné pour extraire les contours et le texte des parcelles à l'aide de données annotées qui correspondent à environ 1/3 d'une feuille de carte parmi les 27 planches de la ville de Venise. Cette annotation correspond à environ 800 parcelles.

Cette méthode a aussi été utilisée pour extraire d'autres couches informationnelles telles que le réseau viaire, les puits et les canaux. L'analyse de ces éléments n'est pas discutée dans cette contribution.

Les bordures extraites sont ensuite utilisées pour calculer les géométries parcellaires auxquelles on fait correspondre les segments de textes. Ces segments de texte sont transcrits par un réseau neuronal récurrent convolutif qui produit une chaîne de caractères lorsqu'on lui donne un segment d'image contenant du texte (Shi, Bai et Yao 2017). Le réseau est entraîné à partir des échantillons d'identifiants provenant des archives vénitiennes et sur des numéros générés synthétiquement avec la base de données MNIST (LeCun, Cortes et Burges 1998). 


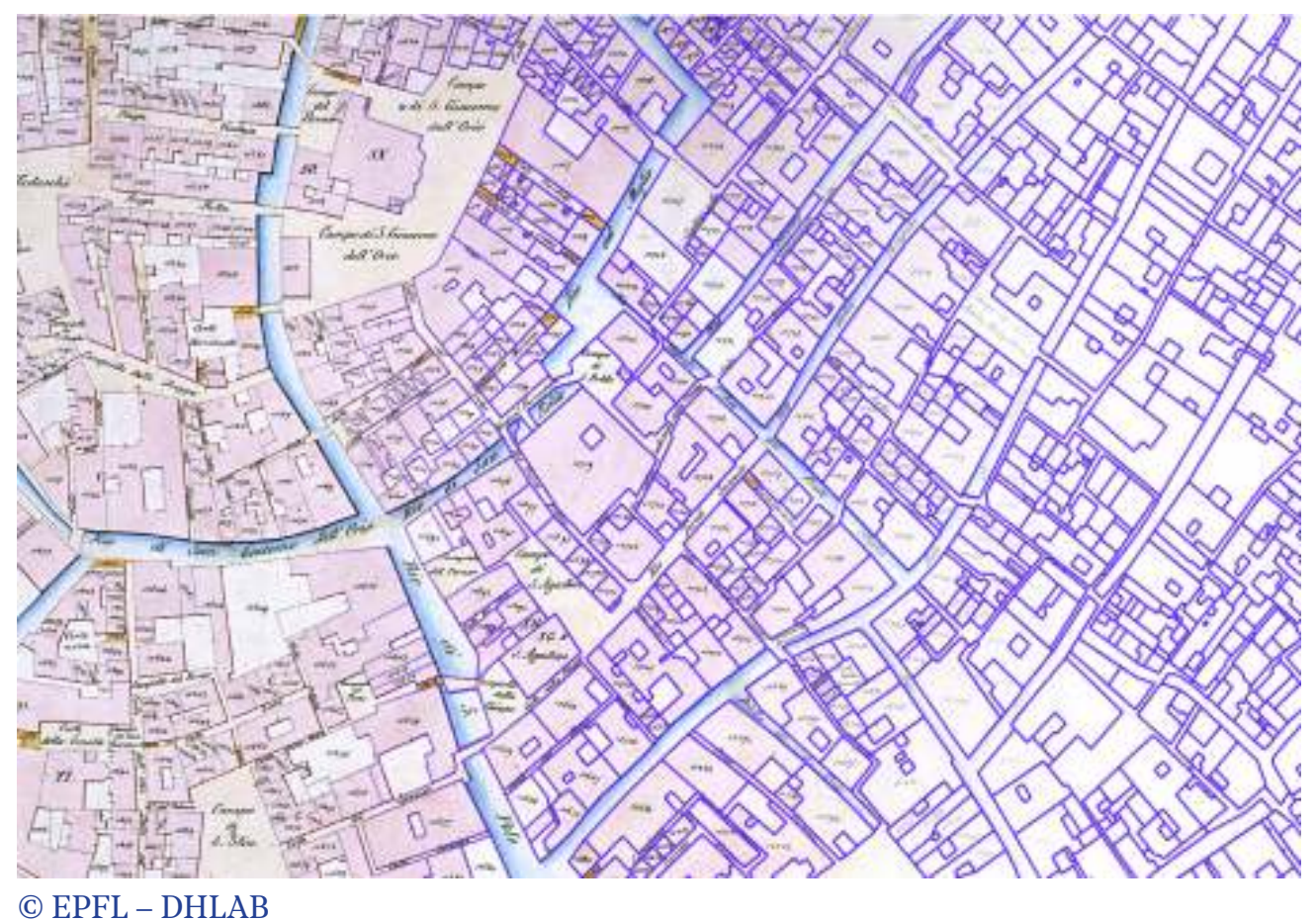

La performance de l'extraction géométrique est mesurée par un calcul d'IoU (intersection over union) comparant les géométriques extraites avec 17 ooo géométries annotées. Pour une valeur seuil de IoU $>0,5$, le niveau de rappel est de $94 \%$ et la précision de $55 \%$. Cela signifie que plus de 9 géométries sur 10 ont pu être extraites automatiquement. Le résultat plus faible en matière de précision s'explique essentiellement par des extractions plus imprécises sur le réseau viaire et les canaux.

Concernant la transcription des numéros de parcelles, la précision obtenue est de $92 \%$ et le rappel de $50 \%$. Les résultats détaillés sont présentés et discutés dans Ares Oliveira et al. (2019). Cette méthode effectue donc une extraction automatique de la géométrie parcellaire et attribue un numéro parcellaire aux géométries. Elle permet d'obtenir un fichier vectoriel au format GeoJSON (format ouvert pour les données géospatiales simples utilisant la norme JSON, JavaScript Object Notation). Dans ce fichier, les géométries des planches ont été identifiées et associées comme attribut ID de la parcelle. Le fichier GeoJSON résultant peut ensuite être importé dans un système d'information géographique, comme QGIS, et peut être traité et exploité dans d'autres formats, tels que shapefile. Une correction manuelle des formes, des ajouts, des oublis et des erreurs de numéros peut ensuite être effectuée dans le logiciel d'information géographique. 


\section{Les registres des états des sections}

Les sept registres sont composés de 15000 pages et plus de 23000 entrées. Toutes ces entrées ont été transcrites manuellement dans un document au format Excel (XLS), ensuite exporté au format CSV, qui garde la même structure tabulaire. Cette extraction aurait aussi pu être effectuée de manière automatique et vérifiée, mais pour la présente étude, l'ensemble de la transcription a été fait et contrôlé à la main pour garantir une meilleure confiance dans l'interprétation des résultats. La redocumentation tabulaire est naturelle, car la source est elle-même un tableau. En outre, les entrées faisant référence à d'autres entrées (c'està-dire comportant les mots "suddetto ", " susdit ", ou " come sopra ", " comme ci-dessus") ont été normalisées afin d'intégrer le texte manquant. Une fois transcrites, les informations peuvent être liées aux géométries extraites précédemment en utilisant le numéro de parcelle, qui est commun. Ces informations peuvent donc être également ajoutées à la table des attributs de chaque géométrie dans le fichier GeoJSON.

\section{La vérification des extractions par la méthode des contraintes}

Les données extraites par ces deux procédés, l'extraction automatisée et corrigée des géométries sur le plan cadastral et la transcription manuelle des registres, comportent inévitablement des erreurs. Ces dernières peuvent venir du procédé d'extraction, qu'il soit fait par une machine ou par un humain, mais peuvent également trouver leur source dans le document lui-même. En effet, en raison de sa taille, la production d'un cadastre suscite immanquablement des erreurs. Toutefois, le fait que le cadastre suive un ensemble de règles précises dans sa construction permet d'établir un ensemble de contraintes qui peuvent être utilisées pour vérifier la cohérence et la complétude des données.

Trois contraintes ont été utilisées pour vérifier la cohérence de l'extraction :

1. Les deux extractions (carte et registre) doivent contenir la suite de numéros de 1 à 15700 ou au minimum les mentionner.

2. Il ne doit y avoir aucun numéro de parcelle en double sur la carte et aucun doublon dans les couples de numéro de parcelle et de subalterno dans le registre.

3. Tout numéro de parcelle présent sur la carte doit se retrouver dans le registre, et vice versa.

Cet ensemble de contraintes a permis de vérifier et de corriger la plupart des données issues de l'extraction. Le processus général consiste à identifier les cas où les contraintes ne sont pas respectées puis à les corriger, ou bien à marquer un cas comme une exception. Le procédé est ensuite répété afin de vérifier que les corrections n'ont pas introduit de nouvelles violations des contraintes et ce jusqu'à ce qu'il n'y ait plus de violations, mais uniquement des exceptions. Ces exceptions correspondent donc à des violations qui ne peuvent être corrigées, du moins pas uniquement avec la source utilisée. De manière générale, ce processus permet donc de vérifier l'exhaustivité des données et de voir où l'information manque. 
Nous allons maintenant passer en revue les différentes violations de contraintes qui n'ont pu être corrigées et nous évaluerons pour finir l'extraction réalisée. Il faut noter qu'une partie des données n’a pas encore été évaluée. Il s'agit des éléments sur la carte qui ont pour identifiant parcellaire des lettres et qui correspondent à des bâtiments religieux ou publics. Ces éléments correspondent à un petit nombre de parcelles (moins de 150) et il n'existe pas de contraintes simples pour assurer leur exhaustivité. En l'état, seules les parcelles numériques sont donc prises en considération dans la discussion.

Les violations de la première contrainte concernent les numéros manquants. Pour le registre, entre 1 et 15700 , un seul numéro, le 4273, n'est pas mentionné dans le document, comme on peut le voir sur la figure 7 . De plus, 14 numéros sont mentionnés mais décrits comme manquants et ne correspondent à aucune entrée. Pour la carte, à l'exception des 14 numéros précédents, 10 numéros manquent. Deux d'entre eux n’apparaissent pas sur la carte. Les 8 autres sont certainement présents sur le document d'origine, mais ne peuvent être lus de manière fiable à cause de l'état de conservation de la carte.

FIGURE 7. IMAGE DE LA PARCELLE 4273 SUR LE MAPPALE ET LE RENVOI DANS LE SOMMARIONE
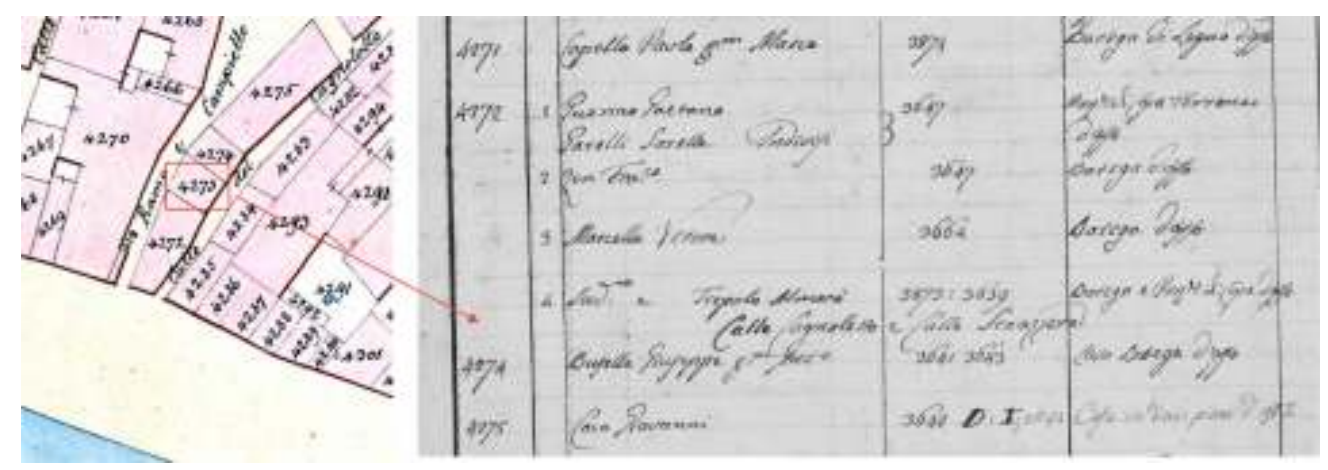

(c) EPFL - DHLAB

La deuxième contrainte vérifie qu'il n'y ait pas de doublons. En effet, chaque entrée et parcelle doivent pouvoir être identifiées de manière unique dans les documents. Aucun doublon n'existe dans les registres, à part des doublons de lettres. Toutefois, de nombreux doublons sont présents sur la carte. Ils peuvent être séparés en trois catégories. La première correspond aux parcelles à cheval entre deux feuilles du cadastre : le numéro étant reporté sur chaque feuille, il est présent plusieurs fois. La deuxième correspond à des parcelles divisées qui sont indiquées avec des fractions (par exemple " 9042 1/2») : dans plusieurs cas, la fraction a été omise sur le plan. Enfin, la troisième catégorie de doublons correspond à des erreurs lors de l'écriture des numéros sur la carte, souvent avec des chiffres proches. Au final, la quarantaine de violations de cette contrainte a pu être résolue en regardant les descriptions des doublons dans le registre et en les faisant correspondre au bon numéro.

La troisième contrainte assure la correspondance entre la carte et les registres. Cette contrainte est particulièrement utile pour retrouver des numéros de parcelles qui correspondent à des divisions de parcelle, comme expliqué dans la contrainte précédente, et qui manqueraient dans une des deux extractions. Cette contrainte a permis de confirmer 
l'absence d'une deuxième parcelle qui est présente sur la carte mais non dans le registre, et de deux parcelles supplémentaires présentes dans le registre mais non sur la carte.

En utilisant ces contraintes et en ne considérant que les parcelles ayant des numéros pour identifiants, seules 12 parcelles existant dans le document n'ont pas pu être liées aux géométries. En prenant en compte toutes les parcelles, lettres comprises, sur les 15851 parcelles du registre, 15795 ont été placées sur la carte. Cela correspond à 23428 entrées sur les 23352 entrées retranscrites, c'est-à-dire 99,67\%. Il est important de noter que seules les correspondances ont été corrigées et que les géométries sont pour la plupart issues de l'extraction automatique.

Le procédé décrit dans la partie précédente nous permet d'obtenir automatiquement dans un système SIG des géométries parcellaires, ainsi que le contenu brut des entrées correspondantes dans le registre. Afin de pouvoir réaliser une analyse plus fine des catégories, il est nécessaire de procéder à une extraction de certaines informations présentes dans le texte transcrit. Quatre genres d'informations ont été extraits : les catégories de la parcelle, le statut légal de propriété, la présence ou non d'une sous-division de la parcelle et la mention d'un ou plusieurs étages. Les informations ont été extraites de manière inductive, c'est-à-dire en partant des données et en créant une liste de catégories et de mots-clés leur correspondant. Pour chacune de ces catégories et chacun de ces motsclés, les entrées pertinentes ont été affinées manuellement afin d'éliminer tout faux positif.

Dans les 8605 champs uniques que constituent les descriptions des catégories fonctionnelles de parcelles, 72 catégories ont été identifiées. Les catégories apparaissant plus de 500 fois sont présentées dans le tableau 1. La plupart des catégories ne sont donc que peu présentes et les 6 catégories du tableau sont représentatives de la plus grande partie des données.

TABLEAU 1. CATÉGORIES EXTRAITES MENTIONNÉES PLUS DE 500 FOIS

\begin{tabular}{|l|l|}
\hline Catégorie & Nombre \\
\hline Casa (maison) & 17738 \\
\hline Bottega (boutique) & 3288 \\
\hline Corte (cour) & 2753 \\
\hline Magazzino (entrepôt) & 1572 \\
\hline Sottoportico (sous-portique) & 795 \\
\hline Orto (jardin potager) & 620 \\
\hline Autres catégories & 1809 \\
\hline
\end{tabular}

(C) EPFL - DHLAB 


\section{Analyse}

\section{Agencement multifonctionnel de la ville}

Considérons tout d'abord l'analyse de l'agencement des fonctions de la ville, c'est-à-dire l'agencement urbain observé non pas du point de vue de la géométrie et de l'espace (urban layout, Martin et March 1975), mais des fonctions (multi-functional layout, Concina 1982). Nous avons vu comment les rédacteurs du document devaient classer des objets urbains selon des catégories spécifiques, puis appliquer la fiscalité correspondante. La question du classement n'est pas totalement nouvelle, elle apparaît déjà dans les protocadastres de l'Ancien Régime, mais, dans ce contexte méthodologique renouvelé, il y a un besoin d'uniformité (Concina 1989). L'intention de l'administration française est de discrétiser l'espace urbain des fonctions, pour permettre un classement univoque. En ce qui concerne la qualité du bien, les éléments numériquement les plus importants du point de vue fonctionnel sont associés aux droits d'utilisation de chaque parcelle. Les droits d'utilisation se divisent eux-mêmes entre propriétés à usage personnel et propriétés louées. Lors de la création de la base de données, la fonction a été extraite et séparée du droit d'utilisation. Comme nous l'avons vu, le nom du locataire n'est pas important pour la définition de la taxe et n'apparaît donc dans aucun registre.

La première analyse concerne la récurrence d'une mention spécifique ou les mentions de chaque élément individuel. Cette première approche ne nous renseigne pas sur les rapports entre les fonctions et ne nous donne pas de détails liés à la spatialisation de l'information, mais documente les fonctions définies par les rédacteurs et l'agencement général de la ville.

Si l'on considère les fonctions individuelles (tableau 1), la maison (casa, 17738 mentions) est l'élément statistique le plus pertinent, suivie de la boutique (bottega, 3 288), de la cour (corte, 2 753) et de l'entrepôt (magazzino, 1 572). Ces trois éléments caractérisent la présence de l'espace habité, de l'espace réservé au commerce de détail, très souvent en association avec d'autres catégories, et d'un espace ouvert, le plus souvent commun entre les parcelles (cour intérieure). L'entrepôt, parcelle à vocation de stockage, occupe une place importante dans les statistiques. Suivent des sous-portiques (sottoportici, 795) qui possèdent une caractérisation non seulement architecturale, mais très souvent aussi fonctionnelle, accueillant les bancs amovibles des marchands : c'est pourquoi ils sont mentionnés dans la source. Les jardins potagers (orti, 620) et les jardins (giardini, 214) sont les fonctions permettant de définir spécifiquement les espaces verts à des fins différentes : l'un non cultivé et l'autre à des fins de culture et donc d'approvisionnement alimentaire de la ville. Les fonctions structurelles spécifiquement connotées d'un point de vue architectural incluent également l'appartement (appartamento, 175), le local (locale, 120), la voûte (volta, 115), le palais (palazzo, 103) et la pièce (stanza, 71). Ces entités définissent en effet différentes typologies architecturales qui caractérisent la résidence et la vente. L'ap- 
partement est une sous-unité d'une parcelle, dénotant le plus souvent une fragmentation du bâti. Dans certains sestieri, et dans certaines zones en particulier, cela explique la présence de parcelles à développement assez élevé et fragmentées par de nombreux appartements, caractéristiques d'une densité de logements importante et probablement de faible valeur économique. On peut également citer la pièce (stanza) parmi ces entités. C'est évidemment l'unité minimale réservée à l'habitation.

Le local (locale) identifie un lieu à usage multiple, mais non résidentiel, probablement destiné au stockage temporaire d'objets ou de marchandises. La voûte (volta) est souvent associée à la catégorie boutique (bottega) et caractérise certains lieux de la ville, notamment les mezzanines sous les voûtes des arcades - c'est le cas du Rialto et de la place Saint-Marc -, ce qui démontre une spécificité de cette dernière. L'approche structurelle et fonctionnaliste met également en évidence certains indicateurs de l'espace de l'Ancien Régime tels que les fours (forni, 36 mentions), les tavernes (osterie, 29), les casernes (caserme, 23), les hospices (ospizi, 17), les marchands de vin (vendivino, 13), les auberges (locande, 10), les nettoyeurs à sec (tintorie, 10), les raffineries (raffinerie, 9), les chantiers de construction (cantieri, 8), les prisons (carceri, 6), les fourneaux (fornaci, 4). Il y a aussi certaines caractéristiques spécifiques des fonctions vénitiennes telles que le squero (19), où les gondoles et les bateaux sont construits et réparés et qui est la seule structure urbaine que nous pourrions considérer comme vraiment unique. Les maisons de jeux (casini, 5) sont également présentes dans le document.

Cette approche structuraliste de la spatialisation s'enrichit encore si, par une simple interprétation basée sur le nombre d'occurrences, nous analysons avec une granularité plus fine l'association des éléments urbains ou la récurrence croisée des mentions. Dans l'analyse du tuple dans lequel apparaissent deux ou plusieurs fonctions en association, certaines unités architecturales et fonctionnelles spécifiques sont plus clairement définies (figure 8).

Tout d'abord, l'association de casa et de corte est prédominante, avec 2230 mentions, suivie de celle de casa et bottega, avec 2031 mentions. La casa avec magazzino (630 mentions) et la bottega avec corte (349 mentions) structurent également l'espace urbain. 


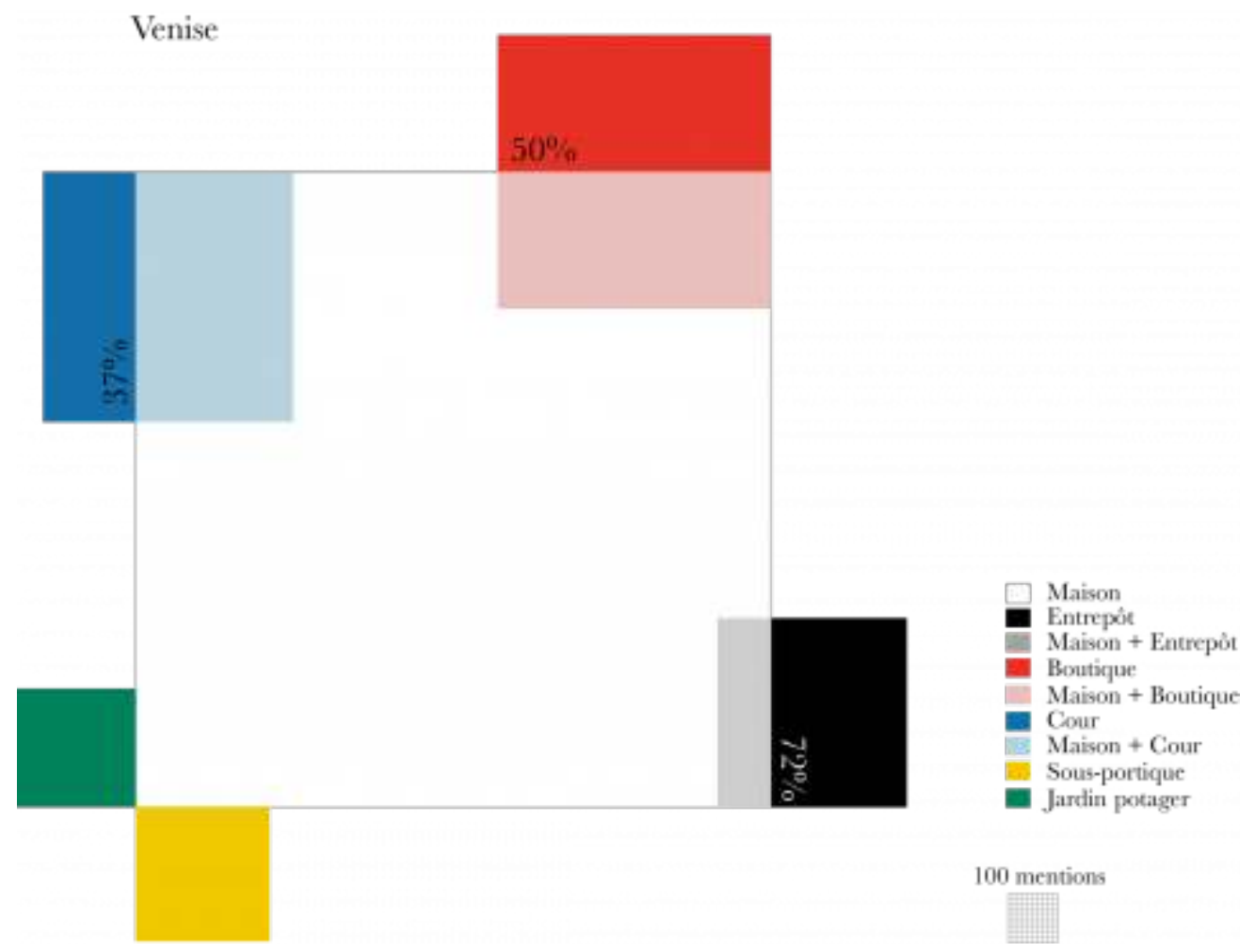

Le diagramme montre de manière synthétique l'agencement complexe des combinaisons de fonctions urbaines. Au centre la macrofonction " maison " est superposée aux fonctions mixtes « maison + boutique ", « maison + entrepôt ", " maison + cour ». L'aire de chaque zone est proportionnelle au nombre de mentions.

\section{(c) EPFL - DHLAB}

Si les droits d'usage s'ajoutent à l'analyse des fonctions, il ressort que le loyer est le droit le plus courant, avec des pourcentages allant de 73 \% à $79 \%$ selon les sestieri. L'utilisation personnelle des biens est minoritaire, avec des pourcentages allant de $5 \%$ à $8 \%$, et cet usage est réservé généralement aux fonctions résidentielles, comme dans le cas des palais ( $p a-$ lazzi). La propriété publique des immeubles commence à prendre forme grâce à une importante campagne d'expropriation, qui se déduit facilement de la présence du mot soppresso/soppressa, c'est-à-dire "supprimé ", mais que nous n'analysons pas ici. Toutefois elle n'est pas encore numériquement importante, puisqu'elle ne représente que $2 \%$ du total de la ville.

Si on analyse les fonctions extraites du cadastre en les combinant avec une analyse spatiale prenant en compte les entités administratives des sestieri (figure 9), on découvre des vocations plus précises. 


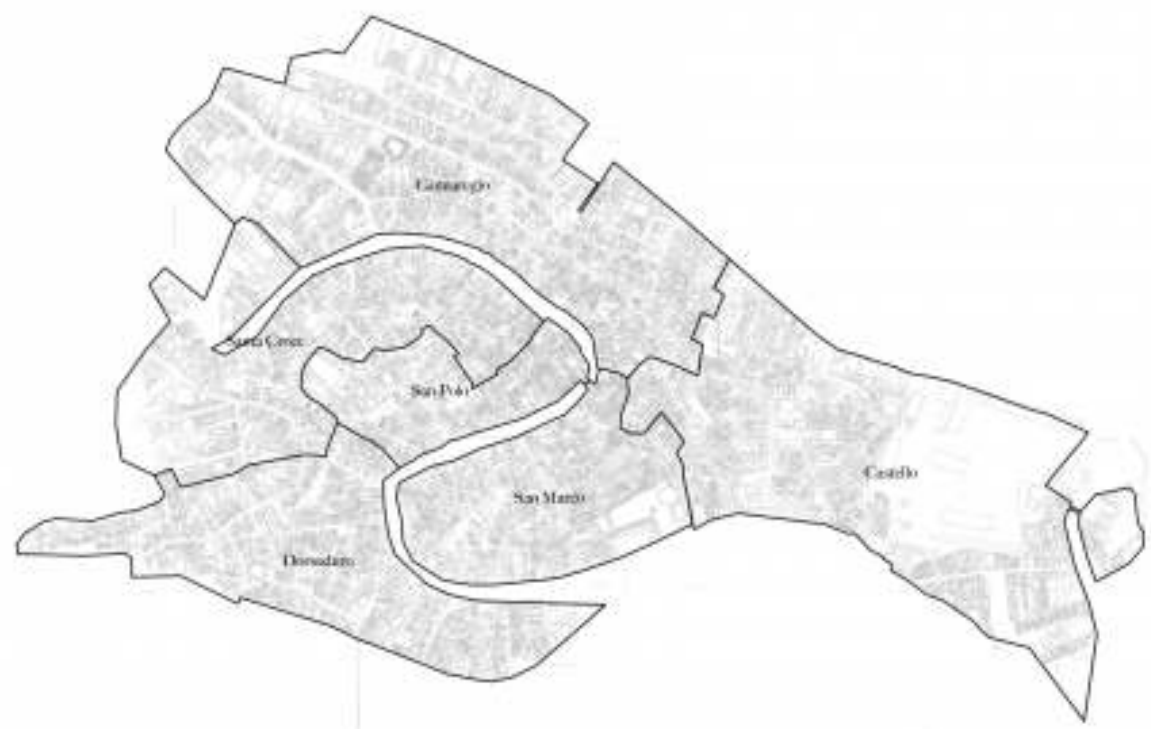

(C) EPFL - DHLAB

La casa (maison) est distribuée presque également dans les sestieri, tandis que son association avec la bottega (boutique) est plus représentée dans les sestieri de San Marco (12,3\%) et Castello (9,8 \%), comme on le voit sur la figure 10. La parcelle composée de la casa (maison) et de la corte (cour), cette dernière étant située à l'intérieur de la parcelle et souvent partagée avec d'autres maisons, est caractéristique du tissu urbain de Santa Croce (16,2 \%) et Dorsoduro (14\%). Les proportions de Castello (7,2 \%) et Cannaregio (4,2 \%) montrent une structure différente, caractérisée par moins d'espaces ouverts appartenant à des parcelles et un parcellaire plus dense. C'est surtout le quartier de San Marco, avec le pourcentage le plus bas $(2,2 \%)$, qui donne à voir une implantation complexe et une densification extrême. 
FIGURE 10. AGENCEMENT MULTIFONCTIONNEL DE CHAQUE SESTIERE, PROPORTIONNEL AU NOMBRE DES MENTIONS DES CATÉGORIES

Cannaregio

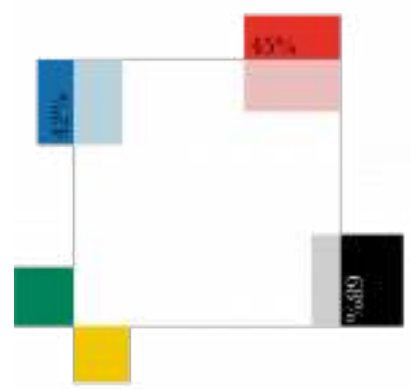

San Marco

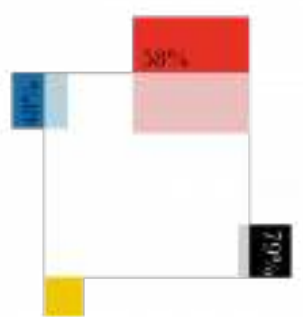

(c) EPFL - DHLAB
Castello

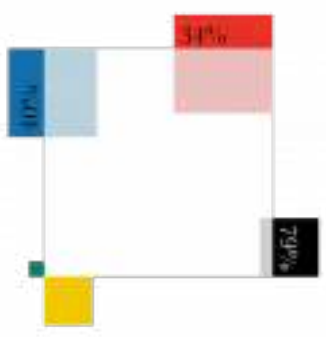

San Polo

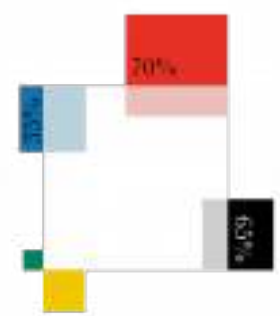

Dorsoduro

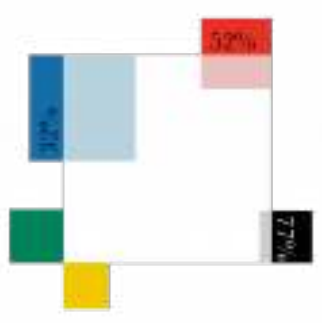

Santa Croce
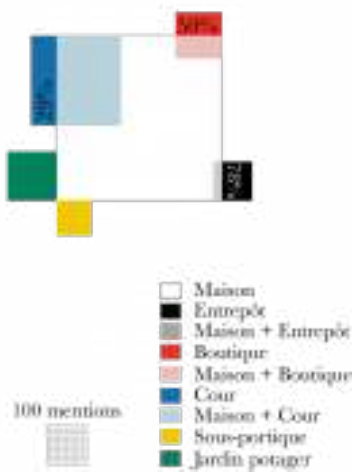

Pour confirmer cette caractéristique, considérons que les jardins (giardini) du sestiere de San Marco occupent o,3 \% du total des mentions, alors que les jardins potagers (orti) ne sont même pas mentionnés. À l'autre extrême, les sestieri de Santa Croce et Dorsoduro se caractérisent respectivement par $22,7 \%$ et $20,7 \%$ de cours (corti) et $6,5 \%$ et $5,3 \%$ de jardins potagers (orti). Les explications sont multiples. 


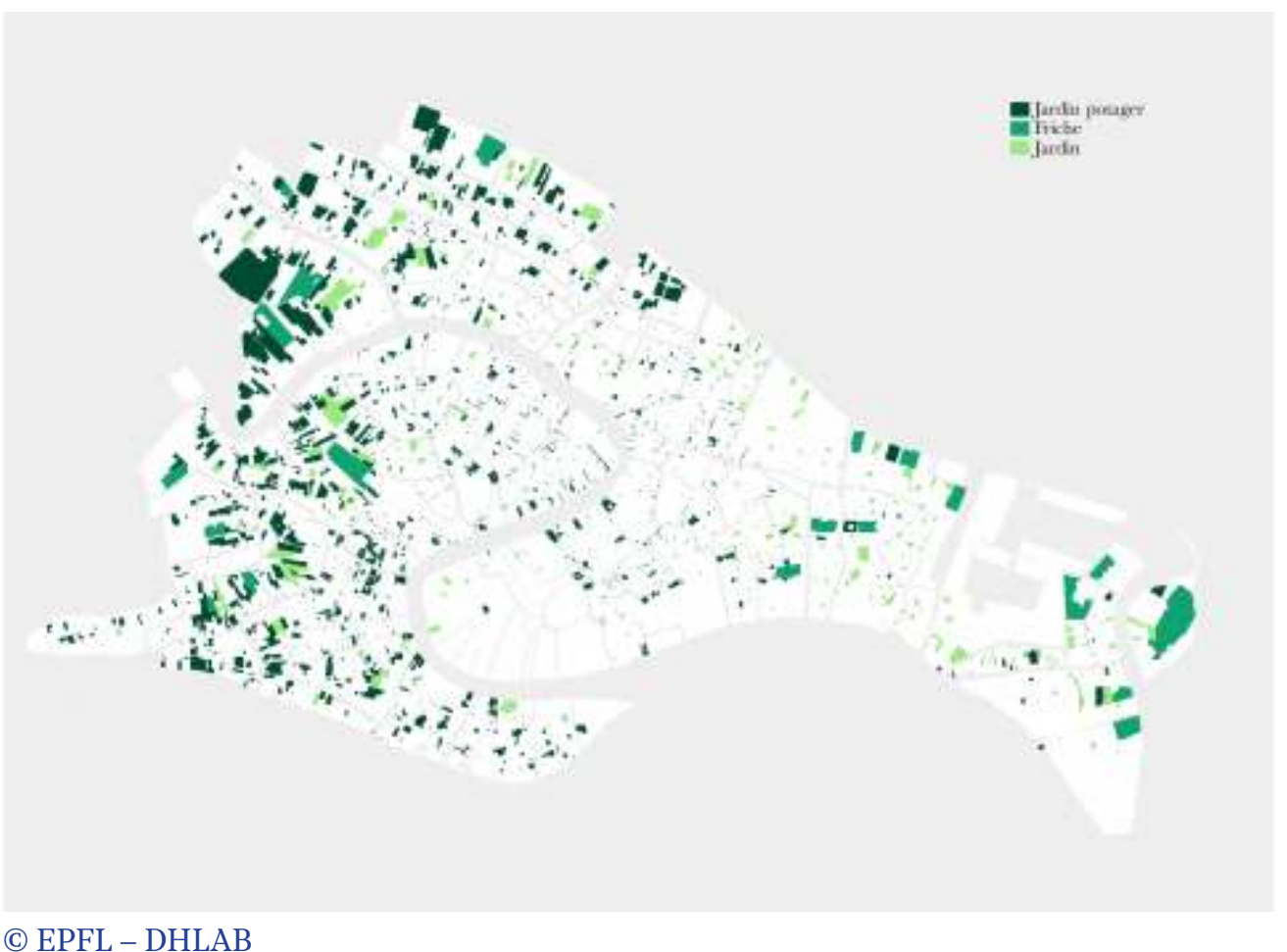

Ces deux sestieri, situés au sud-ouest, se caractérisent depuis des siècles par une implantation résidentielle plus lente, qui s'explique par la remise en état ponctuelle de portions de champs constituées de longues parcelles, lesquelles résultent d'une longue œuvre de bonification (figure 11). Non seulement le tissu immobilier s'est installé plus lentement, mais la présence de grands complexes de couvents a favorisé la présence de larges parcelles de terre cultivée, absentes des quartiers les plus anciens, comme San Marco et San Polo. Dans ces deux derniers, la présence de la catégorie " boutique " (bottega), individuellement et sans association avec d'autres éléments, dénote la vocation commerciale, avec un penchant particulier pour le commerce de détail, qui se développe dans certaines zones spécifiques et sur certains axes routiers majeurs. 


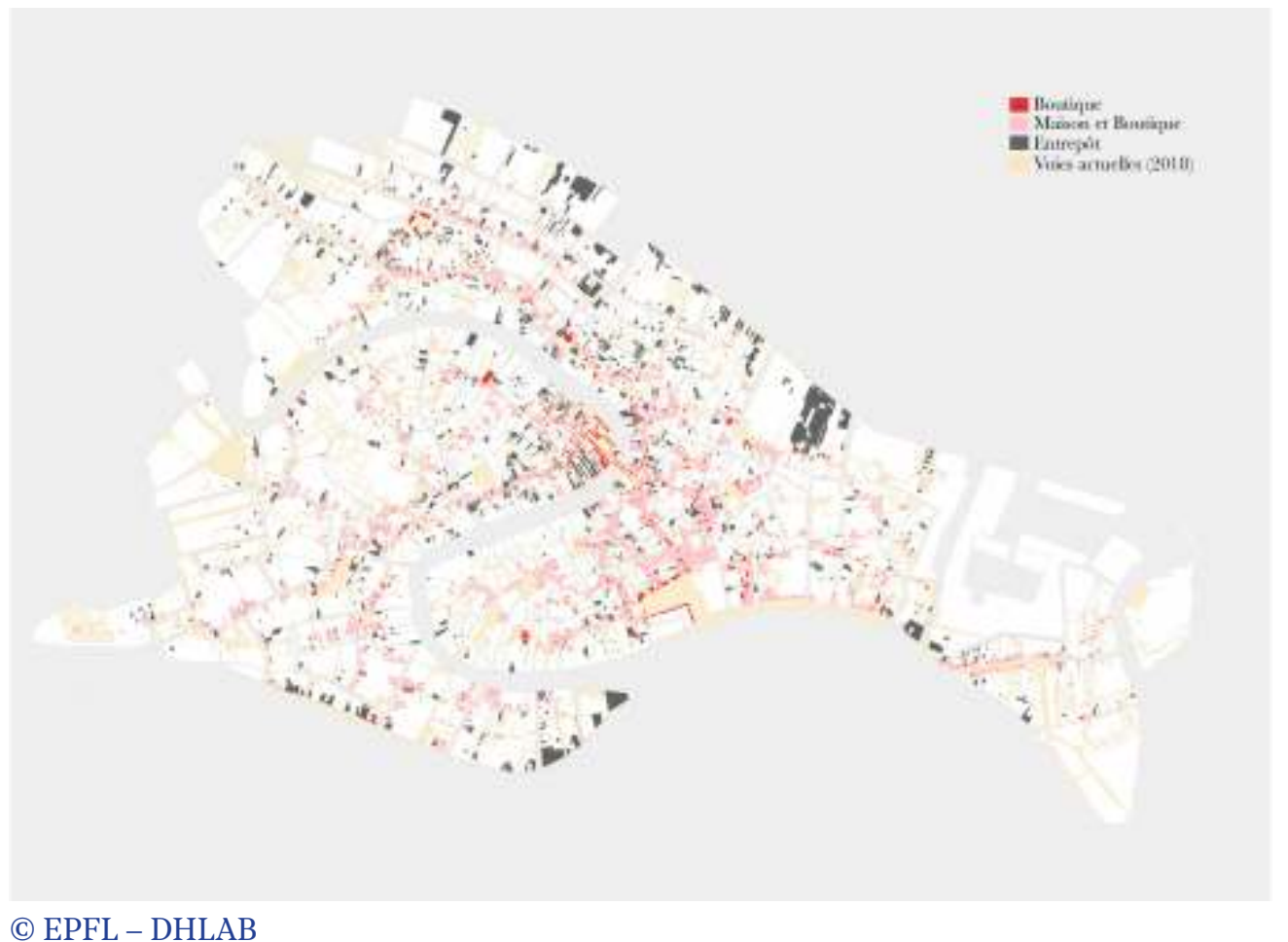

La spatialisation des fonctions qui contiennent une référence explicite au commerce de détail et aux lieux de stockage permet de mettre clairement en évidence différentes spécificités.

La maison (casa) et la boutique (bottega), la boutique mentionnée individuellement et l'entrepôt (magazzino) ont été spatialisés (figure 12). Il apparaît clairement que les parcelles ayant pour fonction la boutique sans autre fonction constituent une spécificité de la zone de la Piazza San Marco et de la zone commerciale du Rialto. En dehors de ces zones, la coprésence de la mention casa et bottega est décrite sur des axes routiers spécifiques : la connexion terrestre entre Rialto et Piazza San Marco, la calle delle Mercerie, ainsi que les plus anciennes calli du sestiere de San Marco, la calle dei Fabbri et la calle della Frezzeria, ainsi que le chemin qui relie le Rialto au cœur du quartier de San Polo, parallèle au Grand Canal. Si on observe la dissémination des boutiques dans le sestiere de Cannaregio, on retrouve une logique intéressante, qui met en évidence un parcours régulier serpentant à travers les calli, et que l'on peut presque superposer à l'actuelle Strada Nuova, dont la percée n'a commencé qu'en 1818, dix ans après la rédaction du plan cadastral (figure 13). 


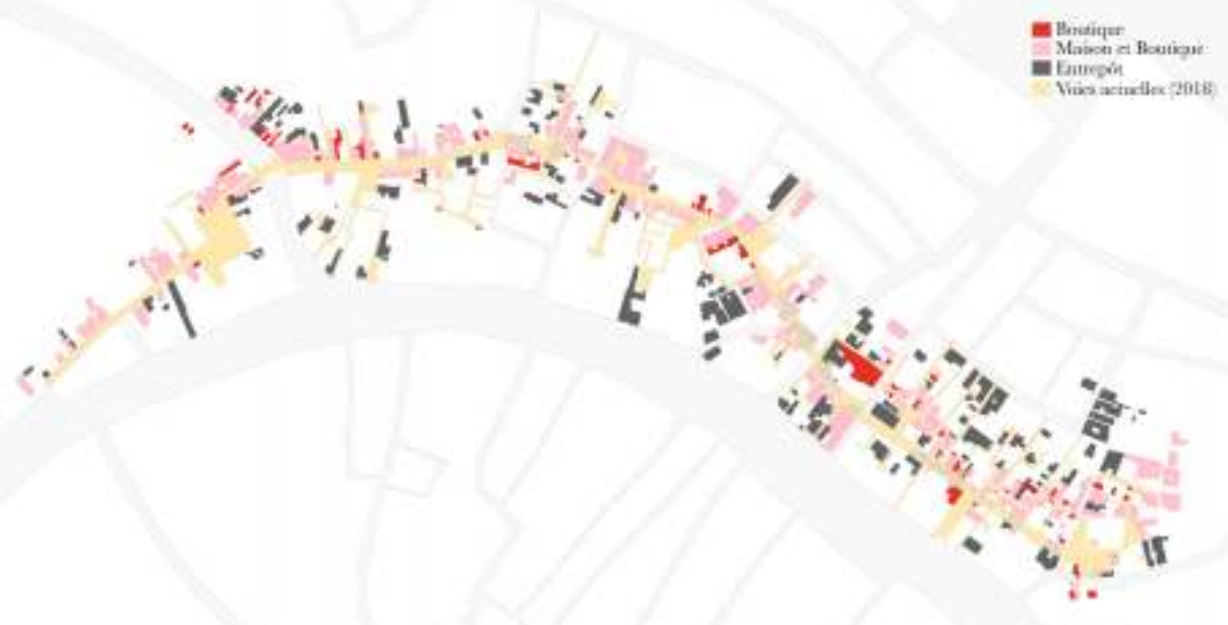

(C) EPFL - DHLAB

Dans le sestiere de Castello, on observe sur les quais du canal Rio de Castello un axe commercial détaché du réseau viaire et situé à l'extrémité de Venise qui desservait le quartier des petits commerces de détail. En 1807, ce canal est comblé et renommé Via Eugenia, comme on peut le voir sur le plan du cadastre. Cette rue deviendra l'actuel Rio Terà Garibaldi, que l'on voit sur la figure 14 . FIGURE 14. SPATIALISATION DU RÉSULTAT DE L'EXTRACTION DES MENTIONS « BOUTIQUE ", "MAISON
+ BOUTIQUE " ET « ENTREPÔT ", AVEC SUPERPOSITION DU RÉSEAU VIAIRE ACTUEL : VUE DÉTAILLÉE DE
LA VIA EUGENIA (AUJOURD’HUI VIA GARIBALDI)

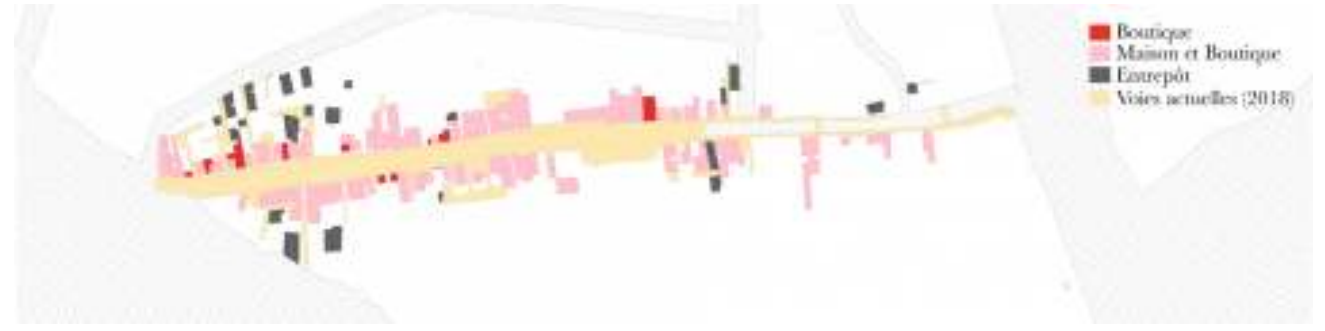

(C) EPFL - DHLAB

Il semble donc que les interventions urbaines ratifient une situation de fait, dans laquelle il existe une densité commerciale spécifique ; dans la plupart des cas, on comble des canaux sur les quais desquels s'étaient installés des commerces en raison de la facilité de l'approvisionnement.

Plus globalement, on peut remarquer qu'il manque un réseau commercial dense capable d'atteindre l'ensemble du tissu urbain de manière uniforme. Si l'on exclut les zones du Rialto et de la Piazza San Marco et l'artère de liaison entre les deux, la calle delle Mercerie, il y a un manque flagrant de planification urbaine pour ouvrir des axes routiers commerciaux desservant le cœur des zones résidentielles, telles que l'interface avec le Grand Canal du sestiere de Santa Croce, l'intérieur de Dorsoduro, les nouveaux quartiers de Cannaregio et le tissu urbain du cœur de Castello. L'analyse spatiale des magazzini (entrepôts) fait émerger clairement leur rôle d'interface entre le cœur de la ville et la lagune. Ils y apparaissent comme la plaque tournante permettant à la fois la communication avec la terre ferme - grâce au réseau fluvial qui amène les matières premières à transformer, comme le bois et les pierres - et 
l'établissement d'un réseau commercial au centre de la ville. Les entrepôts, lorsqu'ils ont une surface considérable et sont accompagnés de terreni (terrains), constituent en effet souvent des plateformes de stockage pour la transformation des matières premières.

Cela est particulièrement visible à la périphérie nord de la ville, dans le grand ensemble d'entrepôts de Castello et Cannaregio, où il y a des entrepôts dédiés aux traitements du bois, souvent associés à des terrains où l'on traite ce précieux matériel de construction, vital pour la ville de Venise. L'interface sud, dans le quartier de Dorsoduro, possède quelques entrepôts, les plus célèbres étant dédiés au stockage du sel, ainsi que des entrepôts accompagnés de la mention casa (maison). Le noyau de Rialto possède également des entrepôts, caractérisés cependant par une surface moins étendue. Il est donc concevable que, dans cette zone, les entrepôts servent de lieux de stockage à court terme, liés au commerce de détail qui caractérise Rialto. Les entrepôts sont presque totalement absents du sestiere de Santa Croce, ce qui montre également son peuplement récent et sa remise en état sans presque aucune activité commerciale ou de transformation.

\section{Carte proxémique}

Les sources administratives peuvent également contenir des informations latentes, non explicitement reconnues et exploitées par ceux qui les ont constituées. À titre d'illustration, la figure 15 montre une représentation de la ville de Venise obtenue à partir des informations des registres cadastraux au moyen d'une série de calculs simples.

FIGURE 15. CARTE PROXÉMIQUE (PROXEMICS MAP) DE LA VILLE DE VENISE SUR LA BASE DU VOISINAGE DES FONCTIONS CADASTRALES

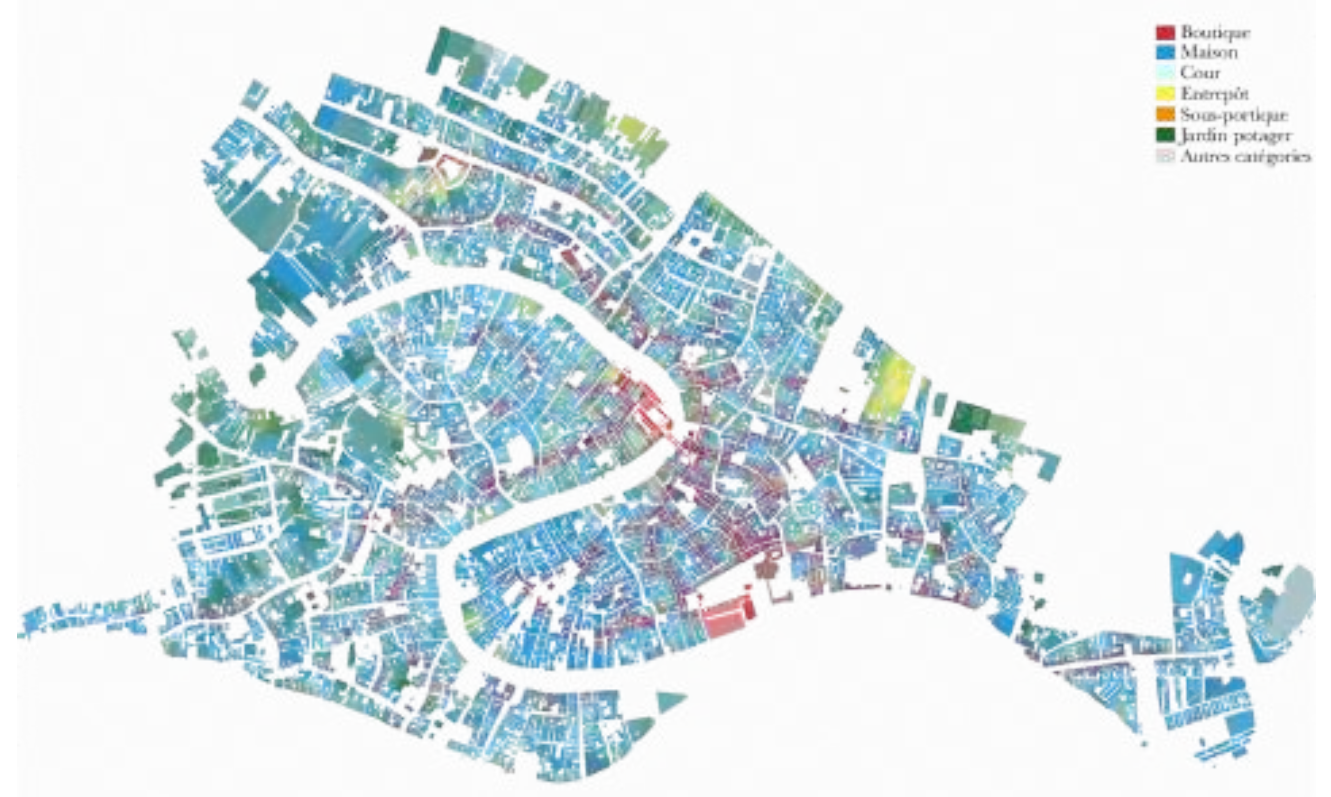

La couleur de chaque parcelle prend en compte les fonctions qui y sont représentées ; cette couleur est pondérée par celles des dix parcelles les plus proches.

(C) EPFL - DHLAB 
- Dans un premier temps, une couleur a été assignée à chacune des six catégories les plus présentes (casa, bottega, corte, magazzino, sottoportico et orto) et le reste des catégories a été associé à la couleur grise.

- Pour initier le calcul, chaque parcelle a ensuite été associée aux couleurs correspondant à sa catégorie, si elle n'en avait qu'une, ou au mélange des couleurs de ses catégories, s'il y en avait plusieurs.

- Enfin, chaque parcelle sur la carte a été associée à une couleur pondérée par celles des dix parcelles les plus proches.

En pratique, avec cet algorithme, une parcelle entourée de botteghe sera de teinte rouge, même si elle n'est pas elle-même une bottega, et une parcelle située dans une zone située à l'intersection entre des parcelles qui ont des catégories différentes, prendra une couleur caractéristique de ce point précis du tissu urbain. Par exemple, une parcelle entourée de botteghe et de case prendra une teinte violette, qui est le mélange pondéré des couleurs. La visualisation a été obtenue en utilisant le fichier GeoJSON contenant les géométries parcellaires, ainsi que les informations sur les catégories. L'algorithme a été implémenté en Python, en utilisant Shapely ${ }^{2}$ pour calculer les distances et GeoPandas ${ }^{3}$ pour gérer les géométries. Le code est disponible en ligne ${ }^{4}$.

La carte proxémique de la figure 15 montre une représentation de Venise selon ce procédé. Cette carte ne montre plus une information extraite directement de la source historique, mais révèle une information qui se trouve à l'état latent dans les données. Le tissu urbain redevient continu et la carte révèle les caractéristiques propres à chaque emplacement au-delà des standards inventés par l'administration napoléonienne.

Sur la carte, les axes commerciaux de couleur rouge sombre, préalablement commentés, sont encore bien visibles comme artères fonctionnelles de la ville, mais la singularité des deux nœuds du pouvoir et du commerce de la ville, le Rialto et la Piazza San Marco, est cette fois évidente, car ce sont les seules zones rouge clair de la carte. L'interface nord de la ville, diaphragme vers l'intérieur du lagon et vers la terre ferme, se distingue de la texture visuelle des quais sud.

Cette carte proxémique met également en évidence les éléments de discontinuité ou de similitude à l'échelle globale. Tout d'abord les limites de la ville ne sont pas toutes identiques et indistinctes, caractérisées uniquement par des entrepôts et des espaces ouverts, mais ont différentes fonctions plus spécifiques.

Dans l'interface nord (en haut de la carte), la gamme de couleur jaunevert distingue clairement les entrepôts et les terrains, associés ensemble, zones réservées à la transformation du bois. C'est le cas du grand périmètre proche de l'église de Santi Giovanni e Paolo, désormais partiellement occupé par l'hôpital civil de la ville. On retrouve les mêmes catégories un peu plus au nord en longeant la zone de la lagune, derrière l'église de la Madonna dell'Orto, dans le quartier de San Marziale et tout près du Ghetto dans le quartier de San Leonardo, où l'on retrouve de très grandes parcelles dédiées aux magazzini et aux terrains de location de bois (affitto da legname). 
Plus au nord, le grand ensemble du district de San Geremia se caractérise par une gamme bleue qui, à y regarder de plus près, représente de grandes parcelles de friches, dont certaines sont en partie plantées de vignes. Dans la continuité du quartier, l'hospice Da Ponte et les frères conventuels de San Giobbe ont les plus grandes parcelles de jardins potagers de la ville.

L'extrémité ouest, dans le sestiere de Santa Croce, est occupée par de longues parcelles de terrain à orientation nord-sud dédiées au blanchiment de la cire - en vert foncé, on y voit aussi des jardins.

La faible présence des commerces, maisons et boutiques démontre la difficulté de créer un tracé routier articulé, comprenant des zones commerciales, et au contraire l'existence d'un tissu urbain étendu à vocation purement résidentielle (en bleu), qui n'est desservi par aucun commerce de détail. C'est en particulier le cas du sestiere de San Marco entre le Rio di San Luca, qui sépare la zone commerciale située entre Rialto et la Piazza San Marco, d'un côté, et le virage du grand canal surplombant le sestiere de Dorsoduro, de l'autre côté.

D’une manière générale, chaque parcelle de la ville est maintenant saisie dans l'unicité de son contexte, qualitativement différente de ses voisines. Les résultats illustrés ici n'exploitent qu'un nombre encore limité d'informations. Une fois densifiée par d'autres éléments synchroniques ou prolongée diachroniquement à partir des informations des cadastres qui, après l'époque napoléonienne, ont poursuivi la documentation systématique de l'information urbaine, la granularité de l'information latente peut se révéler, faisant voir la ville sous des perspectives nouvelles, permettant l'identification de textures, de motifs structurels généraux, mais caractérisant aussi l'unicité de chaque lieu par sa connotation proxémique particulière.

\section{Perspectives}

Cet article a présenté les premiers résultats d'une étude portant sur l'agencement multifonctionnel de la ville et la composition et la distribution des éléments urbains, à partir d'une analyse semi-automatique du cadastre napoléonien de 1808. Il illustre le potentiel et les défis de ce type d'approche. C'est la première fois qu'une telle étude est produite dans le cas de Venise. Nous avons mis en évidence et discuté la structure urbaine, avec les vocations des différents sestieri et les usages des différentes zones de la ville. L'approche de l'analyse granulaire réalisée pour certaines zones, par exemple la Strada Nuova et la Strada Eugenia, a permis de montrer également la dynamique entre les structures préexistantes de la ville et ses modifications ultérieures.

L'interprétation se fonde sur les catégories établies sous l'Empire par l'administration italienne, dans le mouvement de standardisation alors impulsé à l'échelle européenne. Cela doit nous inciter à recontextualiser d'un point de vue technique et politique les cartographies réalisées à partir de ces données. Il s'agira d'abord, dans des travaux futurs, d'interroger la stabilité dans le temps de ces catégories, puis, en changeant d'échelle d'analyse spatiale, de comparer la manière dont les variantes locales de ces typologies ont été déployées, région par région, sur le continent européen. 
Les méthodes numériques d'extraction automatique illustrées ici permettent pour la première fois une redocumentation massive, sous la forme d'une base de données recueillant les informations récoltées par les fonctionnaires de l'Empire. La standardisation et la systématicité des cadastres européens autorisent à envisager un passage à l'échelle, dont l'impact promet d'être important pour les études numériques de l'histoire urbaine. L'arrivée prochaine de bases de données plus denses et plus vastes que toutes celles constituées jusqu'à présent invite à réexaminer systématiquement l'ensemble des résultats antérieurs. Les chercheurs vont en effet bénéficier pour la première fois, non pas d'un nombre intrinsèquement limité d'éléments statistiques sur la structure ou les fonctions du tissu urbain, mais d'un accès complet à la source redocumentée. Cette abondance de données inédites n'est pas sans poser de nouveau défis méthodologiques, mais il ne fait pas de doute que ce changement d'échelle dans la disponibilité des informations aura des conséquences significatives. Dans cette perspective, les données extraites des cadastres européens pourront servir de squelette informationnel et ancrer dans le temps et l'espace des informations issues d'une multitude de sources complémentaires.

\section{Bibliographie}

Antonielli, Livio. 2001. "Venezia nel Regno Italico : un'annessione di "basso profilo" ". Dans Dopo la Serenissima : società, amministrazione e cultura nell'Ottocento veneto, édité par Donatella Calabi, Giuseppe Bonaccorso et Istituto veneto di scienze, lettere ed arti, 123151. Venezia : Istituto veneto di scienze, lettere ed arti.

Ares Oliveira, Sofia, Isabella di Lenardo et Frédéric Kaplan. 2017. « Machine Vision Algorithms on Cadaster Plans ». Communication présentée à Premiere Annual Conference of the International Alliance of Digital Humanities Organizations (DH 2017), Montréal, Canada, 811 août.

Ares Oliveira, Sofia, Isabella di Lenardo, Bastien Tourenc et Frédéric Kaplan. 2019. « A Deep Learning Approach to Cadastral Computing ". Communication présentée à DH 2019 : Digital Humanities Conference, Utrecht, 9-12 juillet.

Ares Oliveira, Sofia, Benoît Seguin et Frédéric Kaplan. 2018. "dhSegment : A Generic Deep-Learning Approach for Document Segmentation». Dans Procceedings of the 16th International Conference on Frontiers in Handwriting Recognition (ICFHR), 7-12. IEEE.

Bianchi, Andrea et Riccio Vangelisti. 2014. « Catasto Lombardo-Veneto del 1853 dei comuni censuari di Bergamo città, Boccaleone, Castagneta, Colognola, Grumello, Redona, Valtesse e Valle d'Astino ». https://territorio.comune.bergamo.it/gfmaplet/?\&map=Catasto1853b/.

Bourguet, Marie-Noëlle. 1988. Déchiffrer la France. La Statistique départementale à l'époque napoléonienne. Paris : Éditions des Archives contemporaines.

Capra, Carlo. 1978. L'età rivoluzionaria e napoleonica in Italia. 1796-1815. Torino : Loescher.

Carassi, Marco et Isabella Massabò Ricci. 1987. « I catasti napoleonici in Piemonte ». Dans Villes et territoire pendant la période napoléonienne (France et Italie). Actes du colloque de Rome (3-5 mai 1984), 96 : 99-120. Roma : École française de Rome.

Chauvard, Jean-François. 2007. « Les Catastici vénitiens de l'époque moderne. Pratique administrative et connaissance du territoire ". Dans De l'estime au cadastre en Europe. L'époque moderne, édité par Mireille Touzery, 421-440. Paris : Comité pour l'histoire économique et financière de la France.

Chiang, Yao-Yi, Weiwei Duan, Stefan Leyk, Johannes H. Uhl et Craig A. Knoblock. 2020. Using Historical Maps in Scientific Studies : Applications, Challenges, and Best Practices. Springer.

Clergeot, Pierre, éd. 2007. Cent millions de parcelles en France. 1807, un cadastre pour l'Empire. Paris : Publi-Topex. 
Concina, Ennio. 1982. Structure urbaine et fonctions des bâtiments $d u X V I^{e}$ au XIX ${ }^{e}$ siècle. Une recherche à Venise. Venise : UNESCO.

Concina, Ennio. 1989. Venezia nell'età moderna. Struttura e funzioni. Venezia : Marsilio.

«Decreto relativo alla formazione del Catasto del Regno, N. 62 ». 1807. Dans Bollettino delle leggi del Regno d'Italia. Parte prima. Dal 1 gennaio al 30 giugno 1807, 193-205. Milano : Reale stamperia.

Deng, Jia, Wei Dong, Richard Socher, Li-Jia Li, Kai Li et Li Fei-Fei. 2009. «Imagenet : A Large-Scale Hierarchical Image Database ». Dans Proceedings of the 2009 IEEE Conference on Computer Vision and Pattern Recognition, 248-255. IEEE.

Doria, Elena. 2014. « La misura della città nel primo Ottocento : i casi di Venezia e Milano. Fonti e strumenti per una lettura comparata della città ». Dans VisibileInvisibile : percepire la città tra descrizioni e omissioni, édité par Salvatore Adorno, Giovanni Cristina et Arianna Rotondo, Scrimm. Catania.

Gioia, Melchiorre. 1808. Tavole statistiche, ossia norme per descrivere, calcolare, classificare tutti gli oggetti di amministrazione privata e pubblica. Milano : Pirotta e Maspero.

He, Kaiming, Xiangyu Zhang, Shaoqing Ren et Jian Sun. 2016. « Deep Residual Learning for Image Recognition ». Dans Proceedings of the IEEE Conference on Computer Vision and Pattern Recognition, 770-778. IEEE.

Kain, Roger J.P. et Elizabeth Baigent. 1992. The Cadastral Map in the Service of the State. A History of Property Mapping. Chicago : University of Chicago Press.

Kaplan, Frédéric et Isabella di Lenardo. 2017. « Big Data of the Past ». Frontiers in Digital Humanities 4. https://doi.org/10.3389/fdigh.2017.00012.

Karabork, H. et E. Aktas. 2008. " A neural network algorithm for vectorization of 2D maps ». Int. Arch. Photogramm. Remote Sens. Spat. Inf. Sci. XXXVII. 473-480.

LeCun, Yann, Corinna Cortes et Christopher J.C. Burges. 1998. « MNIST Handwritten Digit Database ». http://yann.lecun.com/exdb/mnist/.

Locatelli, Andrea. 2003. Riforma fiscale e identità regionale. Il catasto per il Lombardo Veneto (1815-1853). Milano : Vita e pensiero.

Martin, Leslie et Lionel March. 1975. Urban space and structures. London : Cambridge University Press.

Mascilli Migliorini, Paolo. 2011. "Città ». Dans Italia napoleonica. Dizionario critico, édité par Paolo Mascilli Migliorini. Torino : UTET.

Micalizzi, Paolo, Paolo Buonora et Luca Sasso D’Ellia. 2010. « L’informatizzazione della cartografia storica di Roma ». Dans Sistema Informativo Territoriale Archeologico di Roma. Atti del Convegno, Roma, 26 ottobre, édité par Mirella Serlorenzi, 67-76. Roma : Iuno Edizioni.

Noizet, Hélène, Boris Bove et Laurent Costa, éd. 2013. Paris de parcelles en pixels. Analyse géomatique de l'espace parisien médiéval et moderne. Saint-Denis : Presses Universitaires de Vincennes.

Patriarca, Silvana. 1996. Numbers and Nationhood. Writing Statistics in Nineteenth-Century Italy. Cambridge : Cambridge University Press.

Pavanello, Italo, éd. 1981. I Catasti storici di Venezia, 1808-1913. Venezia : Officina.

Repele, Mario, Massimo Rossi et Eugenio Tonetti, éd. 2011. Istruzioni della Direzione centrale del censo ai geometri : incaricati della misura dei territori e formazione delle mappe e dei sommarioni, in esecuzione del $r$. decreto 13 aprile 1807. Officina Topografica.

Romanelli, Giandomenico. 1988. Venezia Ottocento. L'architettura, l'urbanistica. Venezia : Marsilio.

Roncayolo, Marcel. 1987. "Population agglomérée, villes et bourgs en France : réflexions sur les enquêtes de 1809-1811 ». Dans Villes et territoire pendant la période napoléonienne (France et Italie). Actes du colloque de Rome (3-5 mai 1984), 96 : 201-220. Rome : École française de Rome.

Ronneberger, Olaf, Philipp Fischer et Thomas Brox. 2015. «U-Net : Convolutional Networks for Biomedical Image Segmentation ». Dans Medical Image Computing and Computer-Assisted Intervention - MICCAI 2015, édité par Nassir Navab, Joachim Hornegger, William M. Wells et Alejandro F. Frangi, 234-241. Cham : Springer International Publishing. 
Rossi, Fiorenzo. 1998. "Le rilevazioni demografiche nelle terre venete : dalle "anagrafi” veneziane alle statistiche napoleoniche, ". Dans L'area alto-adriatica dal riformismo veneziano all'età napoleonica, édité par Filiberto Agostini, 329-361. Venezia : Marsilio.

Shi, Baoguang, Xiang Bai et Cong Yao. 2017. " An End-to-End Trainable Neural Network for Image-Based Sequence Recognition and its Application to Scene Text Recognition ". IEEE transactions on pattern analysis and machine intelligence 39 (11) : 2298-2304. http://doi. org/10.1109/TPAMI.2016.2646371.

Ughi, Ludovico. 1729. Iconografica rappresentazione della inclita città di Venezia consacrata al Reggio Serenissimo Dominio Veneto. Misc. Mappe, dis. 1234. Archivio di Stato di Venezia.

Uhl, Johannes H., Stefan Leyk, Yao-Yi Chiang, Weiwei Duan et Craig A. Knoblock. 2019. " Automated extraction of human settlement patterns from historical topographic map series using weakly supervised convolutional neural networks ". IEEE Access 8 : 69786996. http://doi.org/10.1109/ACCESS.2019.2963213.

Zucconi, Guido, éd. 2002. La grande Venezia : una metropoli incompiuta tra Otto e Novecento. Venezia : Marsilio.

\section{Notes}

1 Le plugin QGIS RasterBender (créé par Olivier Dalang dans le cadre de ce travail et mis à disposition de la communauté sur GitHub : https://github.com/olivierdalang/RasterBender/) a été utilisé pour améliorer la transformation sur la base de l'alignement des contours du bâtiment.

2 https://shapely.readthedocs.io/en/latest/.

3 https://geopandas.org.

4 https://gist.github.com/raphaelBarman/f027c6a2d1f353271b5987b90994f5af/.

\section{Auteurs}

\section{Isabella di Lenardo}

Collège des Humanités, EPFL, Lausanne, Suisse

Isabella di Lenardo est chercheuse en humanités numériques et histoire urbaine au Collège des Humanités à l'École polytechnique fédérale de Lausanne.

ORCID 0000-0002-1747-9164

isabella.dilenardo@epfl.ch

\section{Raphaël Barman}

Digital Humanities Laboratory, EPFL, Lausanne, Suisse

Raphaël Barman, doctorant en humanités numériques, travaille sur l'extraction automatique d'information dans les sources historiques.

ORCID 0000-0002-0834-177X

raphael.barman@epfl.ch

\section{Federica Pardini}

Digital Humanities Laboratory, EPFL, Lausanne, Suisse

Federica Pardini, doctorante en architecture, étudie la morphologie urbaine à partir des cadastres historiques européens.

ORCID 0000-0001-7201-184X

federica.pardini@epfl.ch

\section{Frédéric Kaplan}

Digital Humanities Laboratory, EPFL, Lausanne, Suisse

Frédéric Kaplan est professeur d'humanités numériques et directeur du Digital Humanities Lab à l'École polytechnique fédérale de Lausanne.

ORCID 0000-0002-6991-5730

frederic.kaplan@epfl.ch 


\section{Droits d'auteur}

(C) (i)

Les contenus de la revue Humanités numériques sont mis à disposition selon les termes de la Licence Creative Commons Attribution 4.0 International. 